\title{
Co-exposure subacute toxicity of silica nanoparticles and lead acetate on cardiovascular system
}

This article was published in the following Dove Press journal: International Journal of Nanomedicine

\author{
Lin Feng ${ }^{1,2}$ \\ Xiaozhe Yang ${ }^{1,2}$ \\ Yanfeng Shi ${ }^{1,2}$ \\ Shuang Liang ${ }^{1,2}$ \\ Tong Zhao',2 \\ Junchao Duan ${ }^{1,2}$ \\ Zhiwei Sun ${ }^{1,2}$
}

'Department of Toxicology and Sanitary Chemistry, School of Public Health, Capital Medical University, Beijing 100069, PR China; ${ }^{2}$ Beijing Key Laboratory of Environmental Toxicology, Capital Medical University, Beijing 100069, PR China
Correspondence: Junchao Duan;

Zhiwei Sun

Department of Toxicology and Sanitary Chemistry, School of Public Health, Capital Medical University, No. 10 Xitoutiao, You An Men, Beijing I00069, PR China

Tel +86 010 839l 1868;

$\mathrm{Tel} / \mathrm{fax}+860108391 \quad 1507$

Email jcduan@ccmu.edu.cn; zwsun@ccmu.edu.cn
Background: The harmful effects following the release of nanomaterials into environment are of great concern today.

Purpose: In this study, subacute effect due to co-exposure to low-dose silica nanoparticles (SiNPs) and lead acetate $(\mathrm{Pb})$ on cardiovascular system was detected in Sprague Dawley male rats.

Materials and Methods: Histopathological and ultrastructural changes of heart, aortic arch and abdominal aorta were detected. Blood routine and blood biochemistry examinations were used to show the changes of blood components. The fibrinolytic and plasmin factors, inflammation-related factors and myocardial-related enzyme in serum were analysised by ELISA and Western blot assay.

Results: Histopathological and ultrastructural examination of heart, aortic arch, and abdominal aorta showed that serious damage occurred in co-exposure group ( $n=6 /$ group). Blood routine examination showed that leukocytosis and thrombocytopenia increased markedly, while changes in the erythrocyte count were not obvious in the co-exposure group. The expression of alanine transaminase (ALT) decreased obviously in co-exposure group, while no significant changes were noted in the expression of aspartate aminotransferase (AST), cholesterol (CHO), triglyceride (TG), high-density lipoproteincholesterol (HDL-C), and low-density lipoprotein-cholesterol (LDL-C) in the co-exposure group on blood biochemistry analysis. In addition, data from ELISA analysis showed that the levels of fibrinolytic and plasmin factors, including thrombin time (TT), prothrombin time (PT), activated partial thromboplastin time (APTT), tissue-type plasminogen activator (t-PA), tissue factor pathway inhibitor (TFPI), and antithrombin III (AT III), were decreased, while those of human fibrinogen (FIB) and D-dimer (D2D) increased significantly in the co-exposure group. Moreover, the myocardial-related enzyme in serum, tested by ELISA, and cardiovascular-related protein expression of atrial natriuretic peptide and brain natriuretic peptide, tested by Western blot assay, was increased in the heart. Furthermore, the expression of inflammation factors such as C-reactive protein (CRP), interleukin-6 (IL-6), and tumor necrosis factor- $\alpha$ (TNF- $\alpha$ ) was increased in heart tissue subjected to combined exposure, which was manifested by Western blot assay, while the protein levels of angiotensin II (ANG II) and endothelin 1 were (ET-1) elevated in blood vessels in the co-exposure group.

Conclusion: In conclusion, the major interactions involved in subacute toxicity due to coexposure to low doses of SiNPs and $\mathrm{Pb}$ on cardiovascular system were expected to be additive and synergistic in nature. Co-exposure to SiNPs and $\mathrm{Pb}$ could aggravate the cardiovascular toxicity via endothelial damage, hypercoagulation, and cardiac injury in vivo.

Keywords: SiNPs, Pb, combined exposure, cardiovascular toxicity, in vivo

\section{Introduction}

In recent years, silica nanoparticles (SiNPs) have been widely applied in the delivery of drugs, vaccines, nucleotides, and proteins. However, exposure to SiNPs could also result in injury to various systems, such as respiratory system, cardiovascular system, 
and so on. ${ }^{1,2}$ In addition, SiNPs are the main constituents of atmospheric particulate matter, ${ }^{3}$ while lead $(\mathrm{Pb})$ is another well-known toxic environmental air pollutant derived from anthropogenic activities. ${ }^{4}$ Nanoparticles possess a unique feature wherein heavy metals could be adsorbed on the surface and subsequently result in adverse toxicity. ${ }^{5}$ Concerns about the combined toxicity of atmospheric particulates and pollutants on living organisms are receiving great attention.

Our previous studies showed that SiNPs could pass through the alveolar-capillary barrier into systemic circulation leading to inflammatory response, nitric oxide (NO)/ nitric oxide synthase (NOS) disorder, and endothelial dysfunction. ${ }^{6}$ Currently, $\mathrm{Pb}$ is also an occupational and environmental toxin causing toxicity to numerous systems and leading to physiological alterations. ${ }^{7}$ Epidemiology studies indicated that exposure to even low levels of $\mathrm{Pb}$ was associated with an increased incidence of clinical cardiovascular end points such as hypertension, arteriosclerosis, coronary heart disease, and stroke. ${ }^{89} \mathrm{~Pb}$ could deposit abnormally on the arterial wall and heart valve and cause constructional and functional transformation of cardiovascular system. ${ }^{10}$ Yet, there is no clear evidence to indicate whether co-exposure to SiNPs and $\mathrm{Pb}$ could lead to cardiovascular toxicity in vivo.

Therefore, this study aimed to 1) assess the subacute effects and mechanisms of SiNPs and $\mathrm{Pb}$ co-exposure on cardiovascular system through the examination of morphology, blood biochemistry and blood routine analysis, and evaluation of cardiovascular-related proteins, and 2) indicate the interactions between SiNPs and Pb by the two-factorial analysis. These findings will provide persuasive evidence for safety evaluation of nanoparticles and also for the low-dose combined toxicity on the cardiovascular system.

\section{Materials and methods}

\section{Animals and treatment}

The specific pathogen-free (SPF) level, 6-week-old, male Sprague Dawley rats used in this study were purchased from Animal Experiments Center of Capital Medical University (Beijing, PR China) and maintained for 7 days before being disposed. The SPF levels of food and water were offered ad libitum to the animals. The weights of Sprague Dawley rats were maintained at $200 \pm 20 \mathrm{~g}$ in the stable conditions which included a temperature of $24^{\circ} \mathrm{C} \pm 1{ }^{\circ} \mathrm{C}, 50 \% \pm 5 \%$ humidity, and a 12 -hour light/dark cycle. All the processes and operations during the entire experimental period were performed in accordance with the rules of Animal Care and Use Committee of Capital Medical University with a permit number of AEEI-2016-087.

The Sprague Dawley rats were randomly divided into four groups ( $\mathrm{n}=6$ for each group) and treated as follows: the blank control group received an intra-tracheal instillation of normal saline; the SiNPs group received $2 \mathrm{mg} / \mathrm{kg}$ body weight of SiNPs in a similar manner. The $\mathrm{Pb}$ group received $0.25 \mathrm{mg} / \mathrm{kg}$ body weight of $\mathrm{Pb}$, and the SiNPs and $\mathrm{Pb}$ co-exposure group received $2 \mathrm{mg} / \mathrm{kg}$ body weight of SiNPs and $0.25 \mathrm{mg} / \mathrm{kg}$ body weight of $\mathrm{Pb}$ mixture (Table S1). Many studies included six animals/group for toxicological experiments. ${ }^{11-14}$ The concentration of each pollutant was fixed according to the international safety standards specified by WHO. This subacute toxicity model lasted for 30 days and received intra-tracheal instillation for 10 times before being executed.

After 30 days' treatment, the rats were anesthetized with $10 \%$ chloral hydrate. The tissues and organs of the heart, aortic arch, and abdominal aorta were isolated and weighted accurately. The coefficients of these tissues and organs were calculated as the ratio of tissues (wet weight, $\mathrm{mg}$ ) to body weight $(\mathrm{g})$. Blood samples were drawn from abdominal aorta, and were immediately placed on ice. Similar tissues from each sample were treated with $4 \%$ paraformaldehyde and glutaraldehyde for further pathological observation and electron microscope ultrastructure analysis. After standing for 2 hours at room temperature, blood samples were centrifuged at 2,000 rpm at $4^{\circ} \mathrm{C}$ for 0.5 hours. All samples were separated and stored at $-80^{\circ} \mathrm{C}$ for further analysis.

\section{SiNPs and $\mathrm{Pb}$ preparation and characterization}

The Stöber technique was used to synthesize SiNPs. ${ }^{15}$ About $2.5 \mathrm{~mL}$ of tetraethyl ortho-SiNPs, $4 \mathrm{~mL}$ of ammonia, $50 \mathrm{~mL}$ of ethanol, and $2 \mathrm{~mL}$ of water were mixed appropriately. After constant stirring at $200 \mathrm{rpm}$ for 12 hours at $40^{\circ} \mathrm{C}$, the mixture was centrifuged at $12,000 \mathrm{rpm}$ for 15 minutes at $4^{\circ} \mathrm{C}$. After washing with deionized water for three times and dispersing in deionized water, SiNPs were characterized by transmission electron microscopy (TEM). $\mathrm{Pb}$ was purchased from Sigma-Aldrich (St Louis, MA, USA). A total of 300 particles were recorded randomly, and their size was measured by ImageJ software to calculate the distribution of particles. The hydrodynamic size and zeta potential of SiNPs were detected by Zetasizer (Nano ZS90; Malvern Instruments, Malvern, UK) in distilled water, DMEM, DMEM with $10 \%$ serum, and normal saline.

\section{Histopathological examination}

For pathological studies, the histopathological tests were performed according to the standard laboratory protocols. ${ }^{10}$ The tissues of heart, aortic arch, and abdominal aorta were maintained in $2.5 \%$ glutaraldehyde for 3 hours. They were washed with $0.1 \mathrm{M}$ PBS, embedded in $2 \%$ agarose gel, 
post-fixed in 4\% osmium tetroxide solution for 1 hour, washed with distilled water, stained with $0.5 \%$ uranyl acetate for 1 hour, and finally embedded in epoxyresin. The resin was polymerized for 48 hours at $60^{\circ} \mathrm{C}$. Ultrathin sections were stained with $5 \%$ aqueous uranyl acetate and $2 \%$ aqueous lead citrate, and observed under a TEM (JEOL JEM2100, Tokyo, Japan). Also, parts of those tissues were maintained in 4\% paraformaldehyde until they were embedded with paraffin. Paraffin sections ( $5 \mu \mathrm{m}$ thickness) were prepared with routine methods, stained with H\&E using standard procedures, cleared in xylene, and mounted with cover slips. The ultrastructure was observed under a light microscope.

\section{Measurement of coagulation factors and plasmin}

Coagulation and plasmin functions were evaluated using several indexes. Blood routine examination was used to determine the basic components and their ratio in the blood. Blood biochemical examinations were used to indicate the activity of cardiovascular-related and coagulation-related enzymes in serum. The four indexes of coagulation, which included prothrombin time (PT), activated partial thromboplastin time (APTT), thrombin time (TT), and human fibrinogen (FIB), were detected to manifest endogenous and exogenous coagulation and plasmin dysfunction. All the biochemical parameters were evaluated according to the commercial kits (Nanjing Jiancheng Bioeng Inst., Nanjing, PR China).

\section{Coagulation factors and fibrinolytic index measurements}

The concentrations of blood clotting factors, D-Dimer (D2D), antithrombin III (AT III), tissue-type plasminogen activator (t-PA), and tissue factor pathway inhibitor (TFPI), were determined by ELISA kits (USCN Biotech Company Limited, Beijing, PR China). The ELISA test was duplicated for all serum samples. The absorbance was measured under the microplate reader (Themo Multiscan MK3; Thermo Fisher Scientific, Waltham, MA, USA) at $450 \mathrm{~nm}$.

\section{Myocardial-related protein measurements}

The concentrations of atrial natriuretic peptide (ANP) and brain natriuretic peptide (BNP) which are myocardial-related function factors were determined by ELISA kits (USCN Biotech Company Limited). The ELISA test was duplicated for all serum samples. The absorbance was measured under the microplate reader (Themo Multiscan MK3; Thermo Fisher Scientific) at $450 \mathrm{~nm}$.

\section{Western blot analysis}

Briefly, total protein samples of heart, aortic arch, and abdominal aorta were extracted with the Tissue Protein Rapid Extraction Kit (Dingguo, Beijing, PR China) and were determined with the bicinchoninic acid protein assay (Pierce, Rockford, IL, USA). Equal amounts of lysate proteins (100 mg) were loaded onto SDS-polyacrylamide gels $(10 \%$ separation gels) and transferred to polyvinylidenefluoride membranes (EMD Millipore, Billerica, MA, USA) electrophoretically. After blocking with 5\% nonfat milk in Tris-buffered saline (TBS) for 1 hour at room temperature, the membrane was incubated with angiotensin II (ANG II), endothelin 1 (ET-1), atrial natriuretic peptide (ANP), brain natriuretic peptide (BNP), C-reactive protein (CRP), interleukin-6 (IL-6), and tumor necrosis factor- $\alpha$ (TNF- $\alpha$ ) (1:1,000, rabbit antibodies; Abcam, Cambridge, UK) overnight at $4^{\circ} \mathrm{C}$. After washing with TBS containing $0.05 \%$ Tween-20 (TBST) for three times, the membrane was incubated with a horseradish peroxidase-conjugated anti-rabbit IgG secondary antibody (Abcam) for 1 hour at room temperature. After washing with TBST three times again, the blotted proteins were imaged through the Odyssey Infrared Imaging System (LI-COR Biosciences, Lincon, NE, USA). The gray intensity of proteins were counted using ImageJ software.

\section{Statistical analysis}

Data were expressed as mean $\pm \mathrm{SD}$, and significance was determined by one-way ANOVA in SPSS 23.0 followed by least significant difference test to compare the different treatment groups. The two-factorial analysis was used to indicate the interactions between SiNPs and lead. Differences were considered significant at $P<0.05$. Their marginal means were compared with the profile plots (interaction plots). ${ }^{16,17}$ The interaction types were as follows: 1) additive effect: $F<5, P>0.05$, and $\mathrm{E}_{\mathrm{A} \times \mathrm{B}}>\mathrm{E}_{\mathrm{A}}+\mathrm{E}_{\mathrm{B}} ; 2$ ) synergistic effect: $F>5, P<0.05$, and $\mathrm{E}_{\mathrm{A} \times \mathrm{B}}>\mathrm{E}_{\mathrm{A}}+\mathrm{E}_{\mathrm{B}}$; and 3) antagonistic effect: $F>5, P<0.05$, and $\mathrm{E}_{\mathrm{A} \times \mathrm{B}}<\mathrm{E}_{\mathrm{A}}+\mathrm{E}_{\mathrm{B}} \cdot{ }^{18}$

\section{Results}

\section{Characterization of SiNPs and $\mathrm{Pb}$}

The shape of SiNPs was near-spherical and the particles were well isolated under the TEM (Figure 1A). The hydrodynamic sizes and zeta potentials of SiNPs and $\mathrm{Pb}$ in distilled water and normal saline at different time points are shown in Figure $1 \mathrm{~B}$ and $\mathrm{C}$ and Table S2. Zeta potentials provided quantitative information on the stability of the particles. It was found that the particles dispersed well when the zeta potential was more than $30 \mathrm{mV} .{ }^{19}$ 
A

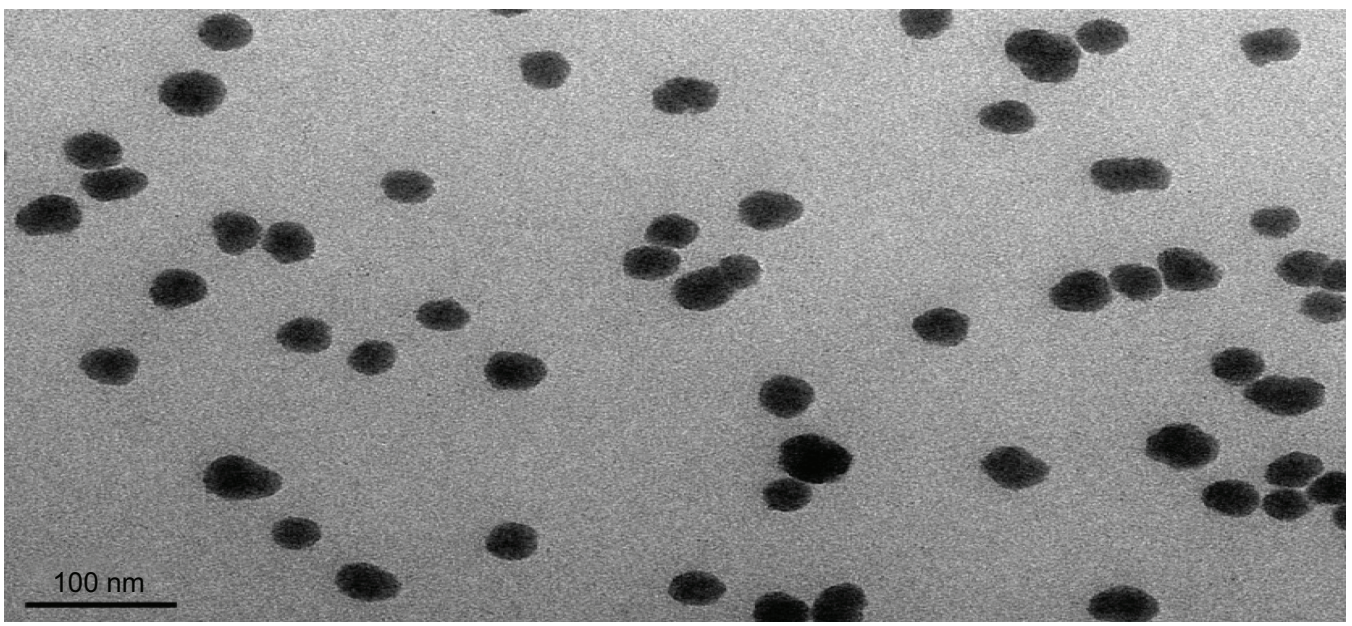

B

Size distribution by intensity

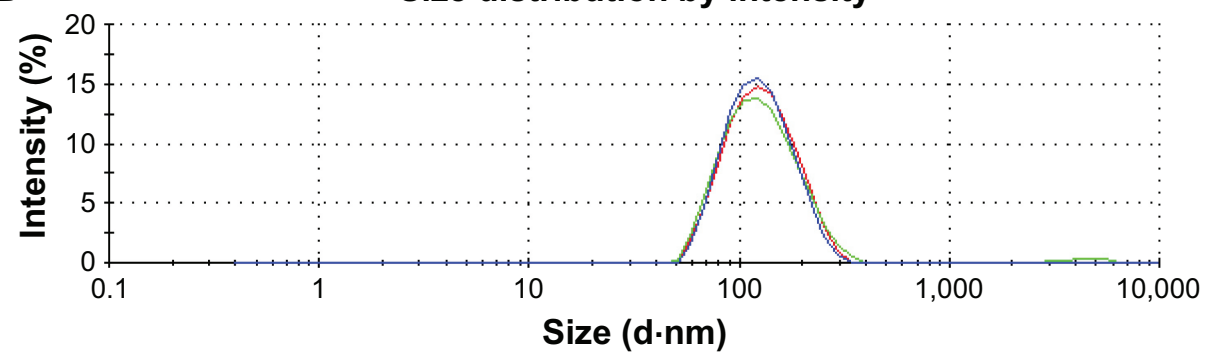

C

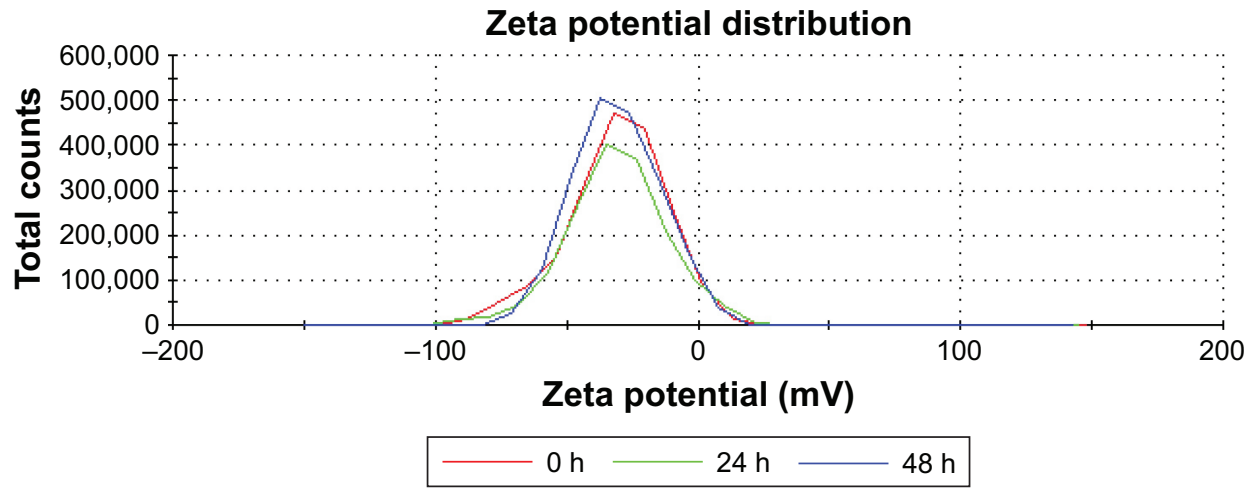

Figure I Characterization of the SiNPs and $\mathrm{Pb}$.

Notes: (A) The TEM image shows spherical and well-dispersed particles. (B) Size distribution by intensity. (C) Zeta potential distribution.

Abbreviations: Pb, lead acetate; SiNPs, silica nanoparticles; TEM, transmission electron microscopy.

The mixture of SiNPs and $\mathrm{Pb}$ exhibited good monodispersity in both distilled water and normal saline. This mixture had an absolute value of zeta potential more than $30 \mathrm{mV}$, which showed good stability.

\section{Histopathological changes in heart}

The electron microscopy image of the heart is shown in Figure 2A-D. In heart tissue, the cardiac muscle fibers were arranged in an orderly manner. The membranes of mitochondria and intercalated disc were integrated, and the density of intima crest was normal in control group.
The orderliness of cardiac muscle fibers and the local vacuolation of mitochondria could be observed in SiNPs alone and $\mathrm{Pb}$ alone groups. In the co-exposure group, the cardiac muscle fibers were disrupted, the mitochondria were condensed, the crest of intima was disappearing, and more collagenous fiber occurred in mesenchyme. The pathological changes in Figure 2E-H showed that the heart was normal in both control and SiNPs groups. The inflammatory cells, swollen necrosis, and serious derangement appeared in the $\mathrm{Pb}$ and co-exposure groups. In our study, cardiotoxicity in co-exposure group was stronger than any single group. 

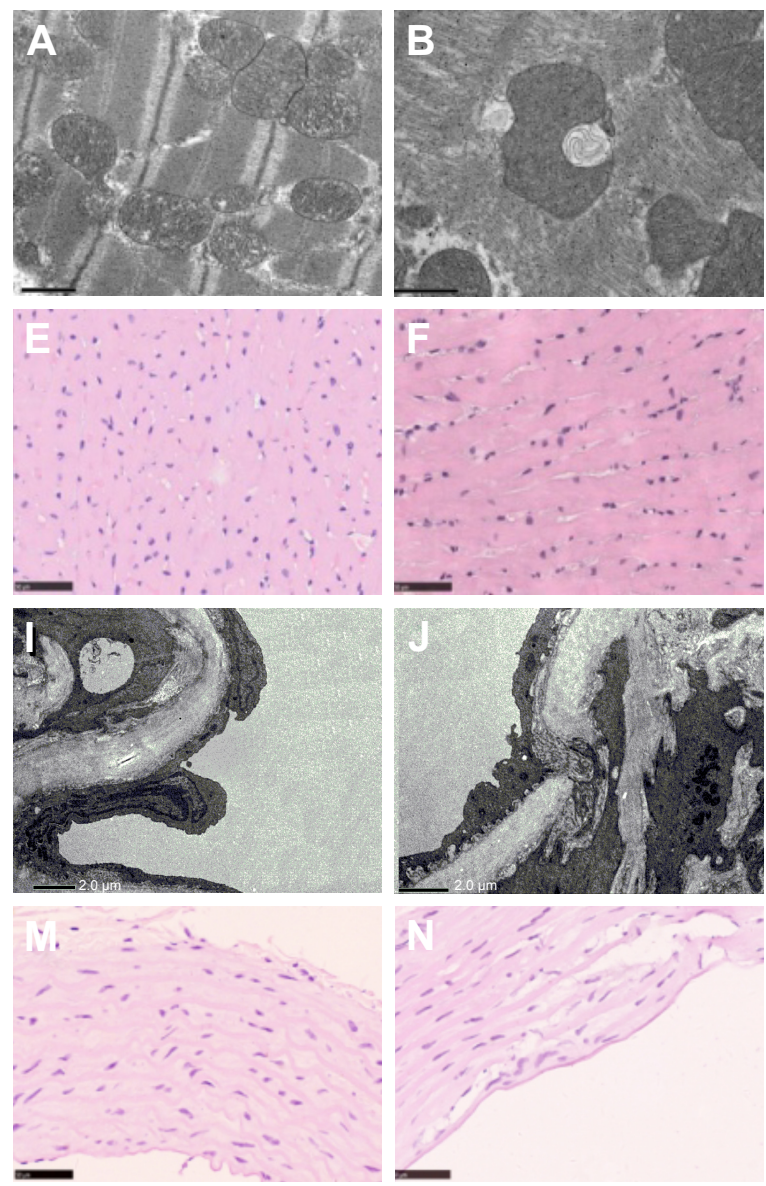
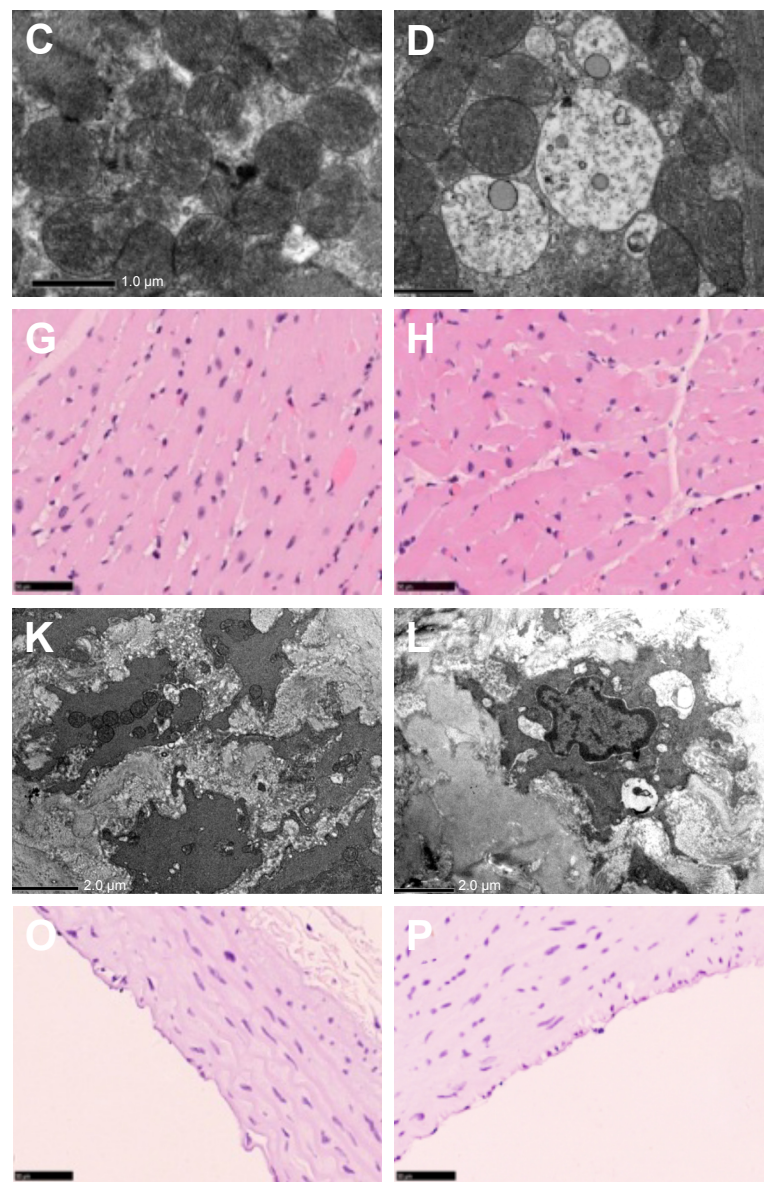

Figure 2 The TEM image of heart in Sprague Dawley rats triggered by SiNPs, Pb, and co-exposure: (A) control group, (B) SiNPs group, (C) Pb group, (D) SiNPs + Pb group. The H\&E image of heart in Sprague Dawley rats triggered by SiNPs, Pb, and co-exposure: (E) control group, (F) SiNPs group, (G) Pb group, (H) SiNPs + Pb group. The TEM image of aortic arch in Sprague Dawley rats triggered by SiNPs, Pb, and co-exposure: (I) control group, (J) SiNPs group, (K) Pb group, (L) SiNPs + Pb group. The H\&E image of aortic arch in Sprague Dawley rats triggered by SiNPs, Pb, and co-exposure: (M) control group, (N) SiNPs group, ( $\mathbf{O}) \mathrm{Pb}$ group, $(\mathbf{P}) \mathrm{SiNPs}+\mathrm{Pb}$ group.

Abbreviations: SiNPs, silica nanoparticles; Pb, lead acetate; TEM, transmission electron microscopy.

\section{Histopathological changes of abdominal aorta}

The electron microscopy images of the abdominal aorta are shown in Figure 2I-L. In vessel tissues, the endothelial cells and basal membrane were integrated in the control and SiNPs groups. The local vacuolation of endothelial cells and the dissolution of nuclear membrane could be observed in $\mathrm{Pb}$ alone group. In co-exposure group, the swelling of mitochondria and exfoliative endothelial cells was observed. The pathological results in Figure 2M-P showed that the abdominal aorta was normal in both control and SiNPs groups, the inflammatory cells were observed, and edema appeared in the $\mathrm{Pb}$ and co-exposure groups. In our study, vessel toxicity in the co-exposure group was stronger than in any of the single-exposure groups.

\section{The blood coagulation function}

In our study, we performed blood routine examination, and evaluated blood biochemical indexes and four coagulation indexes to assess the coagulation function. Blood routine examination indicated the red blood cell (RBC), leukocyte (WBC), and blood platelet (PLT) counts. The WBC indexes showed an obvious increase in co-exposure group than in single-exposure groups, which might be due to possible inflammation in the blood system. The lymphocyte (LYM) and monocyte (MON) count increased obviously in $\mathrm{Pb}(P<0.05)$ and co-exposure groups $(P<0.01)$ when compared with control group (Figure 3A-F). The PLT count decreased in $\mathrm{Pb}$ and co-exposure groups than in control group, which might be due to possible functional variation in coagulation (Figure 3G-I). The RBC count exhibited no obvious changes in different groups (Figure $3 \mathrm{~J}-\mathrm{Q}$ ). In blood biochemistry reports, ALT levels decreased in the co-exposure group than in other groups, while there were no obvious changes in the levels of aspartate aminotransferase (AST), cholesterol (CHO), triglyceride (TG), high-density lipoprotein-cholesterol (HDL-C), and low-density lipoprotein-cholesterol (LDL-C) 
in the serum $(P>0.05)$. Results are shown in Figure 4. The four coagulation indexes including TT, APTT, FIB, and PT were detected in blood serum to manifest the endogenous and exogenous coagulation and plasmin function. The TT and PT levels showed no obvious changes, while the APTT level was decreased and the FIB level was increased with no statistical significance. All the results of TT, APTT, FIB, and PT showed additive interactions $(F=0.016, P=0.901 ; F=0.735$, $P=0.402 ; F=0.369, P=0.550 ; F=0.018, P=0.895$, respectively) between SiNPs and $\mathrm{Pb}$. Results are shown in Figure 5.

\section{Coagulate- and myocardial-related factors}

As shown in Figure 6, we determined the coagulate-related (D2D, AT III, TFPI, and t-PA) and myocardial-related (ANP
A

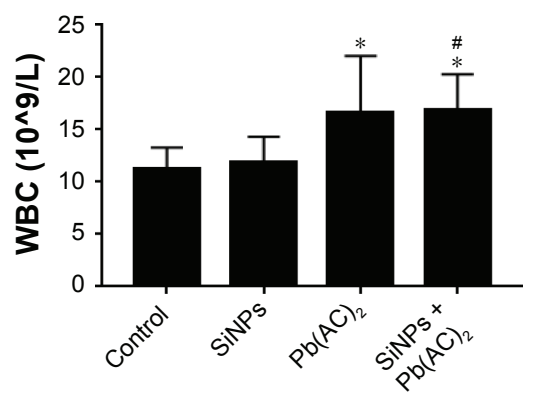

D
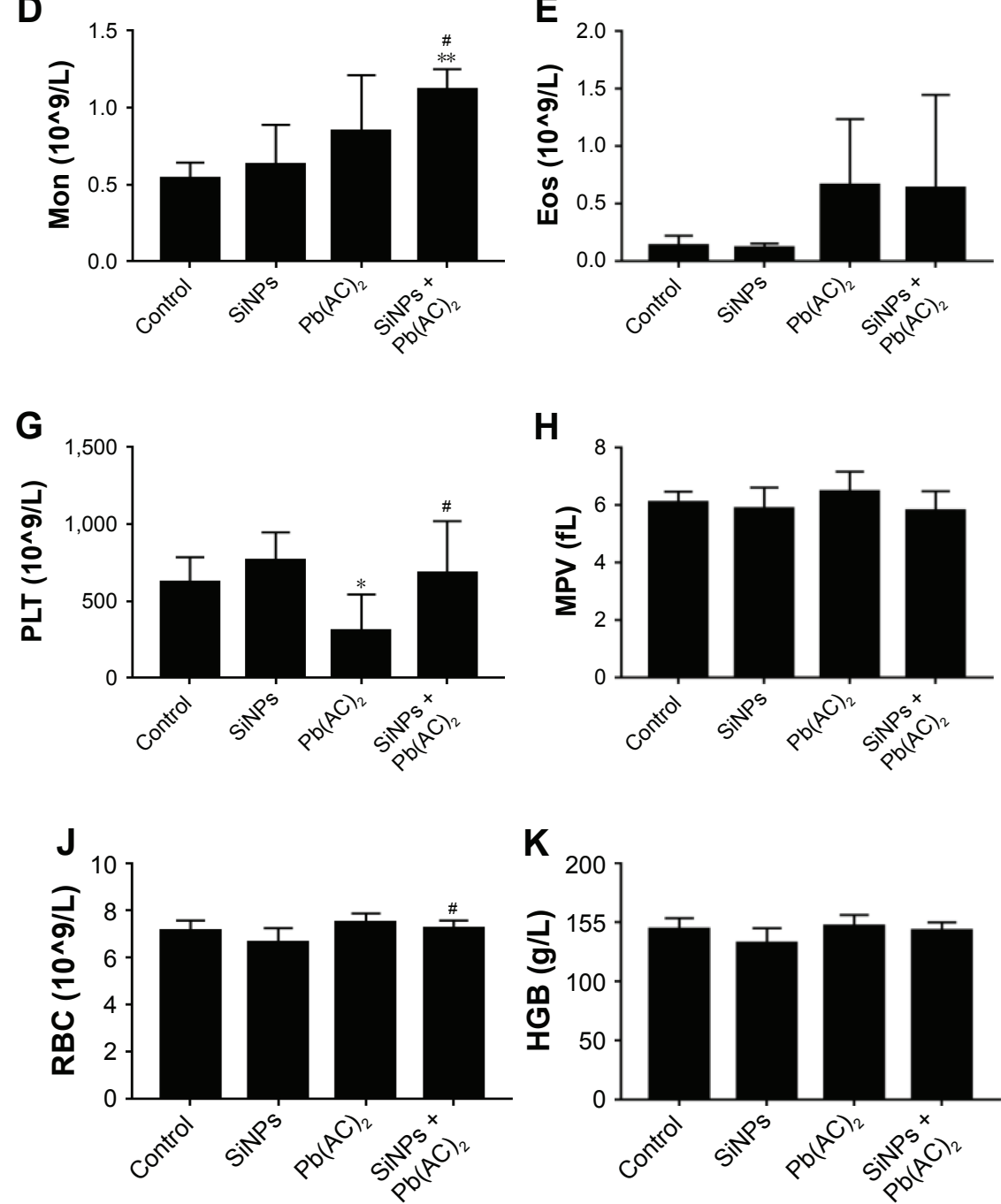

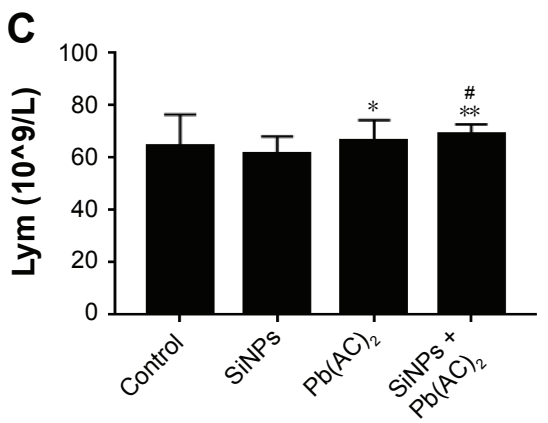

E
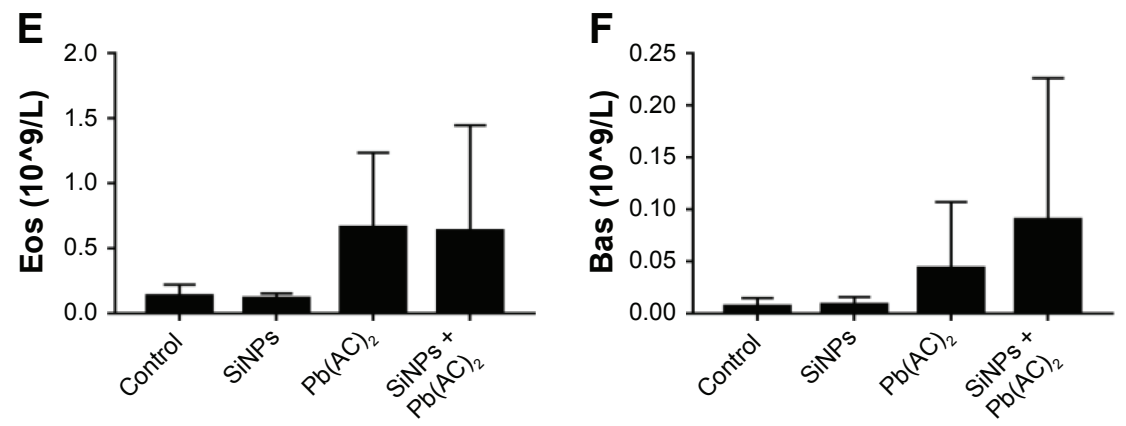

H
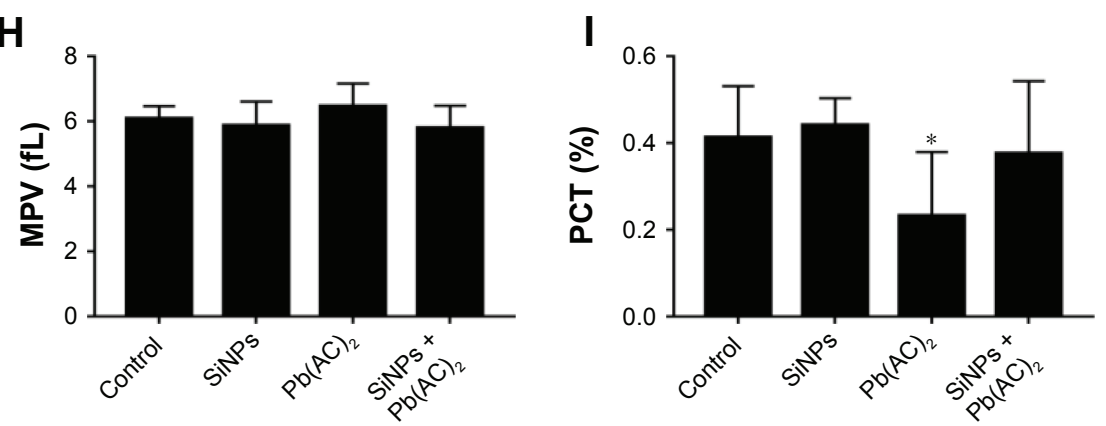

$\mathbf{L}$

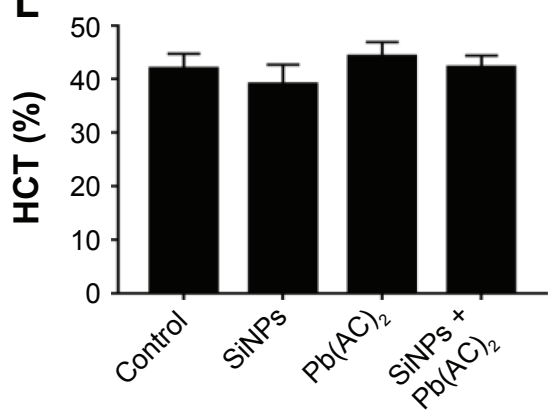

Figure 3 (Continued) 

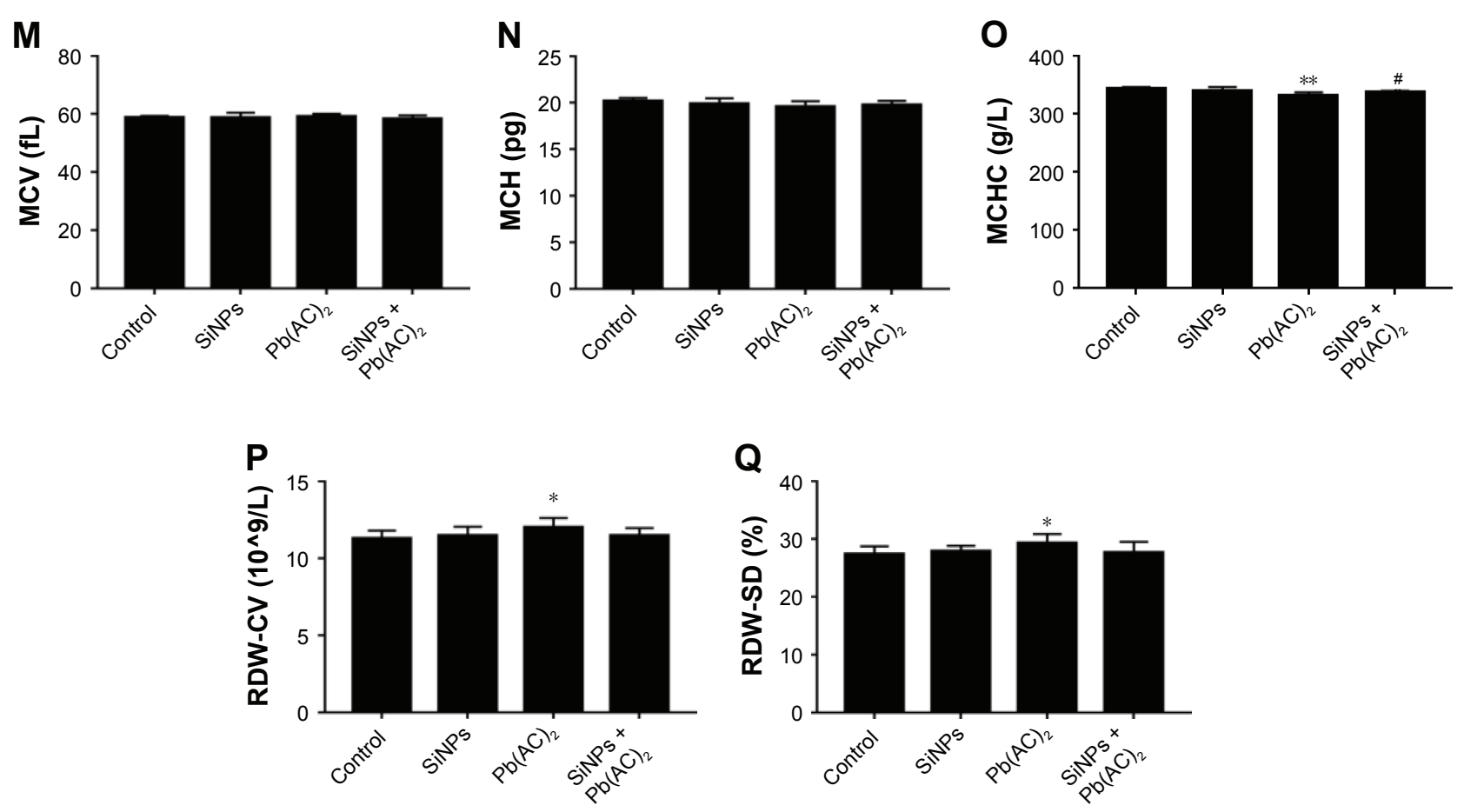

Figure 3 The blood routine examination.

Notes: (A-F) Leukocytes. ${ }^{\# P}<0.05$ when comparing co-exposure group with control and SiNPs groups in WBC (4A); ${ }^{*} P<0.05$ when comparing co-exposure group with control and SiNPs groups in Lym (4C); ${ }^{\# P<0.05}$ when comparing co-exposure group with control and SiNPs groups in Mon (4D). (G-I) Thrombocytes. ${ }^{*}<<0.05$ when comparing co-exposure group with $\mathrm{Pb}$ group in PLT (4G). (J-Q) Erythrocytes. ${ }^{\# P<0.05}$ when comparing co-exposure group with $\mathrm{SiNPs}$ group in RBC (4J); ${ }^{\# P<0.05}$ when comparing co-exposure group with $\mathrm{Pb}$ group in $\mathrm{MCHC}(4 \mathrm{O})$. Data are expressed as mean $\pm \mathrm{SD}$ from six independent experiments. $* P<0.05$, $* * P<0.0 \mathrm{I}$ compared with untreated control, ${ }^{\# P}<0.05$ compared with individual toxicant alone treated group.

Abbreviations: Bas, basophil; Eos, eosinophil; Lym, lymphocyte; Mon, monocyte; Pb(AC), lead acetate; PLT, blood platelet; RBC, erythrocyte; SiNPs, silica nanoparticles; WBC, leukocyte; MPV, mean platelet volume; PCT, plateletcrit; HGB, hemoglobin; HCT, hematocrit; MCV, mean corpusular volume; MCH, mean corpusular hemoglobin; MCHC, mean corpusular hemoglobin concerntration; RDW-CV, red blood cell distribution width-coefficient of variation; RDW-SD, red blood cell distribution width-standard deviation.

and BNP) factors to manifest the cardiovascular function. The results showed that TFPI and t-PA levels were decreased significantly, while D2D level was increased significantly and AT III level decreased to a lesser extent in the co-exposure group when compared to control group. Results of D2D, AT III, and t-PA showed additive interaction $(F=2.229, P=0.151$; $F=1.337, P=0.261 ; F=0.911, P=0.351$, respectively) between
SiNPs and $\mathrm{Pb}$. Results of TFPI showed synergistic interaction ( $F=35.640, P=0.000$ ) between SiNPs and $\mathrm{Pb}$ (Figure 6A-D).

The ANP and BNP levels increased significantly in the co-exposure group than in the control group. When comparing the co-exposure group with the single-exposure group, increase in the levels was found to be significant. Interaction analysis showed additive effect in ANP
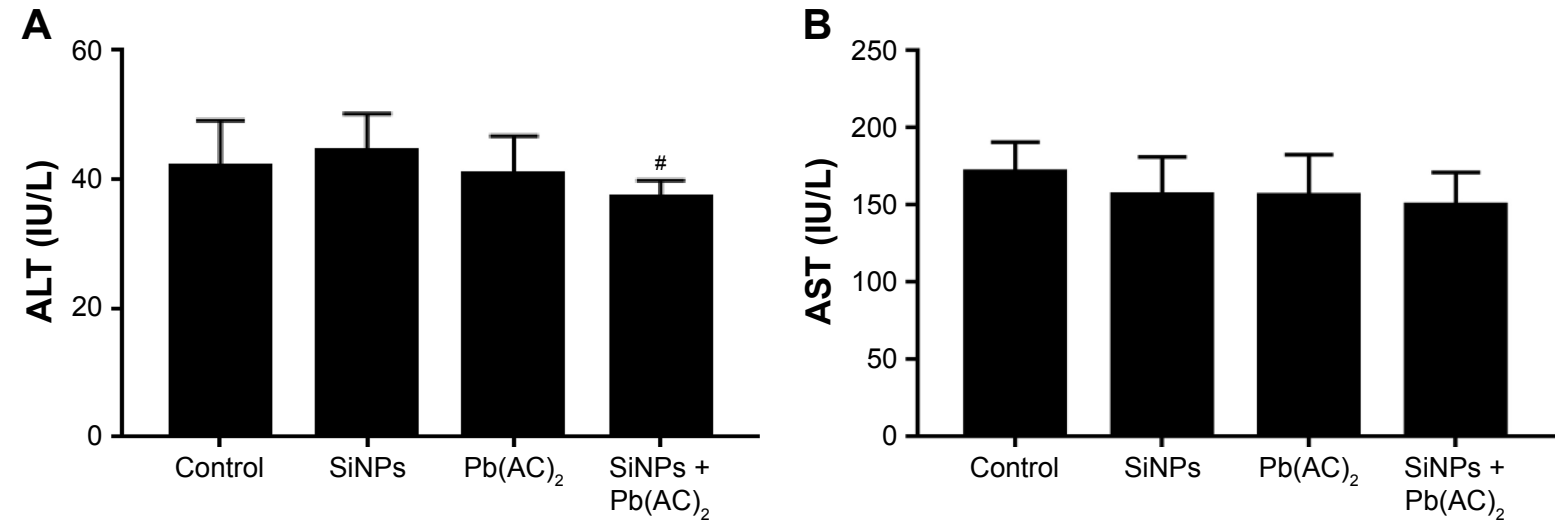

Figure 4 (Continued) 

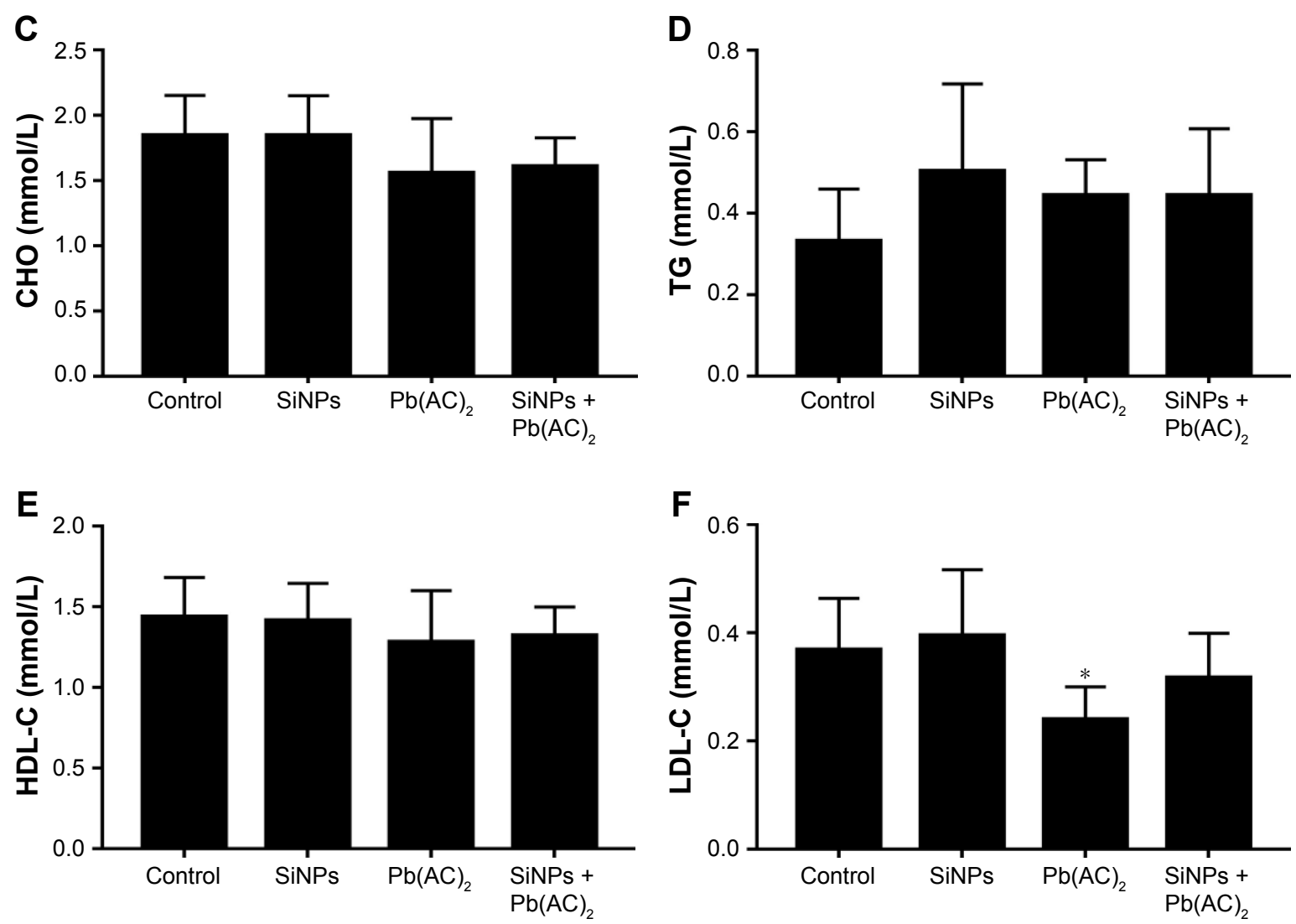

Figure 4 The blood biochemistry examination.

Notes: (A, B) ALT and AST. ${ }^{\sharp P}<0.05$ when comparing co-exposure group with SiNPs group in ALT (5A). (C-F) Serum lipid levels. Data are expressed as mean \pm SD from six independent experiments. $* P<0.05$ compared with untreated control, ${ }^{*} P<0.05$ compared with individual toxicant alone treated group.

Abbreviations: HDL-C, high-density lipoprotein-cholesterol; LDL-C, low-density lipoprotein-cholesterol; Pb(AC), lead acetate; SiNPs, silica nanoparticles; ALT, glutamicpyruvic transaminase; AST, aspartate transaminase; $\mathrm{CHO}$, total cholesterol determination; TG, triglyceride.

factor $(F=1.358, P=0.258)$ and synergistic effect in BNP factor $(F=14.836, P=0.001)$ between $\operatorname{SiNPs}$ and $\mathrm{Pb}$ (Figure 6E and F).

\section{Cardiovascular-related proteins}

To explore the effect on different molecule processes deeply, we detected cardiovascular-related protein levels in the heart and vessels of Sprague Dawley rats. As shown in Figure 7A (myocardial-related), the expression of ANP and BNP was increased after exposure in the combined group, and the inflammation factors such as CRP, IL-6, and TNF- $\alpha$ were also increased after exposure in the combined group. As shown in Figure 7B (vessel-related), the expression of ANG II and ET-1 was increased significantly after exposure in co-exposure group when compared with the control and single-exposure group, which showed strong constriction of blood vessels in the co-exposure group. Overall, co-exposure to SiNPs and $\mathrm{Pb}$ at low doses may cause greater cardiovascular dysfunction and inflammatory response than exposure to single $\mathrm{SiNPs}$ or $\mathrm{Pb}$. The schematic representation of this study is given in Figure S1.

\section{Discussion}

Recent studies have provided adequate evidence regarding cardiovascular toxicity as a result of exposure to the single pollutants like SiNPs or $\mathrm{Pb} .^{20,21}$ The complex mixtures could have interactive effect on the catalytic levels and transcriptional proteins. ${ }^{22}$ Our previous studies have shown adverse toxicity after co-exposure, cardiovascular toxicity in particular. ${ }^{23,24}$ Growing evidence has confirmed the complex composition of ultrafine particles and that their adsorption might alter the individual effects of these pollutants. As for the various interactions among the different pollutants in the air, toxicity resulting from co-exposure to nanoparticles and heavy metal ions could cause more critical effects on cardiovascular system. Therefore, this study could offer a new perspective to explore the cardiovascular toxicity and interactions between SiNPs and $\mathrm{Pb}$.

Generally, from our results (Figure 1 and Table S2) and other studies, the zeta potential of nanoparticles was more stable in distilled water than in normal saline. Higher ionic strength of the buffer and lower affinity of surface bounders were related with the worse zeta potential reliability. ${ }^{25}$ 
A

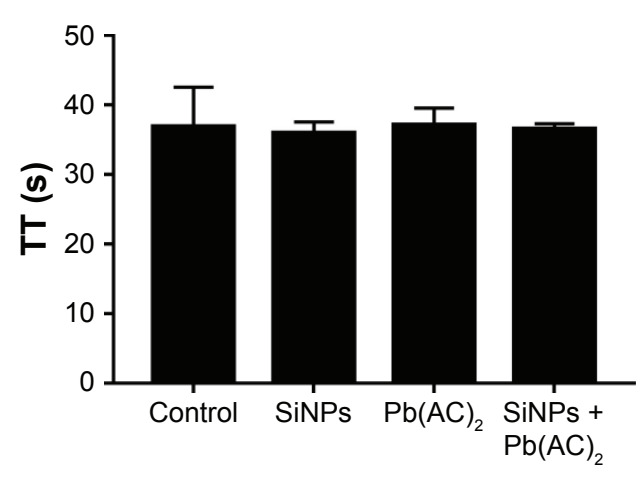

B

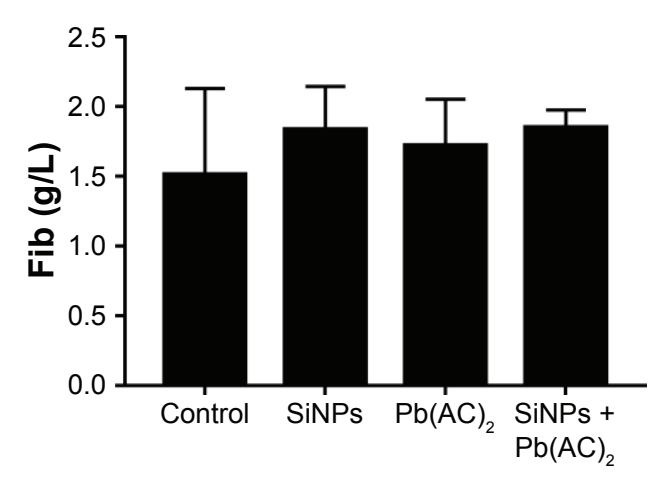

C

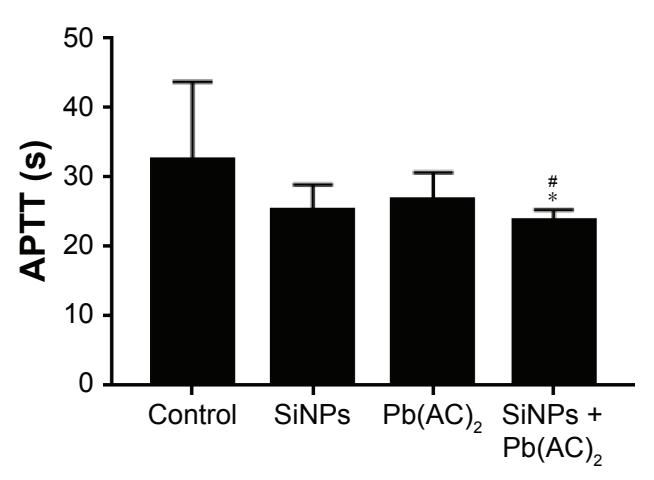

D

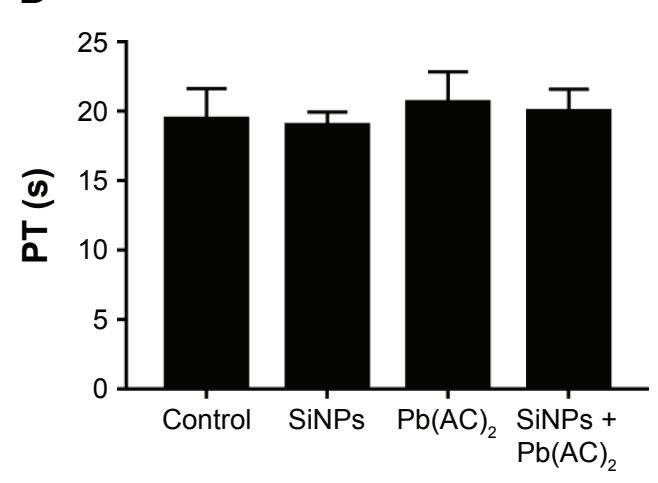

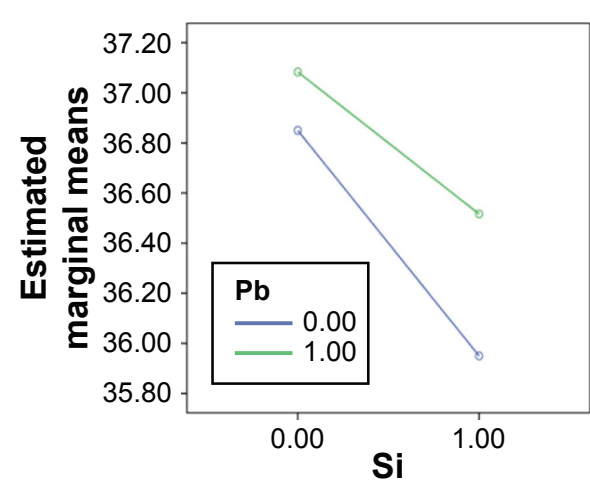
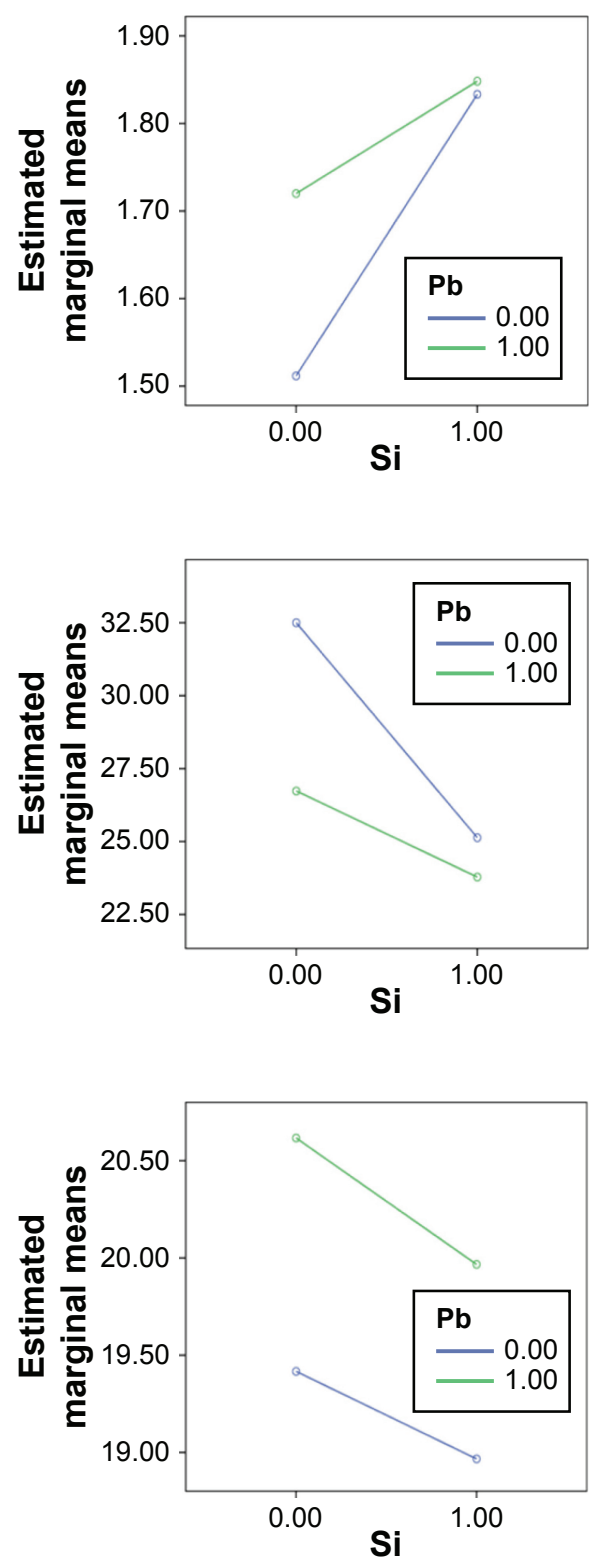

Figure $\mathbf{5}$ The four coagulation tests.

Notes: $(\mathbf{A})$ TT results showed additive interaction between $\mathrm{SiNPs}$ and $\mathrm{Pb}(\mathrm{AC})_{2}(\mathrm{~F}=0.016, \mathrm{P}=0.90 \mathrm{I})$; $(\mathrm{B}) \mathrm{FIB}$ results showed additive interaction between $\mathrm{SiNPs}$ and $\mathrm{Pb}(\mathrm{AC})_{2}$ $(F=0.369, P=0.550)$; (C) APTT results showed additive interaction between SiNPs and $\mathrm{Pb}(\mathrm{AC})_{2}(F=0.735, P=0.402)$, ${ }^{*} P<0.05$ when comparing co-exposure group with control group in APTT; (D) PT results showed additive interaction between SiNPs and $\mathrm{Pb}(\mathrm{AC})_{2}(F=0.018, P=0.895)$. Data are expressed as mean $\pm \mathrm{SD}$ from six independent experiments. ${ }^{* P}<0.05$ compared with untreated control, ${ }^{*} P<0.05$ compared with individual toxicant alone treated group.

Abbreviations: APTT, activated partial thromboplastin time; FIB, human fibrinogen; $\mathrm{Pb}(\mathrm{AC})_{2}$, lead acetate; PT, prothrombin time; SiNPs, silica nanoparticles; TT, thrombin time. 
A

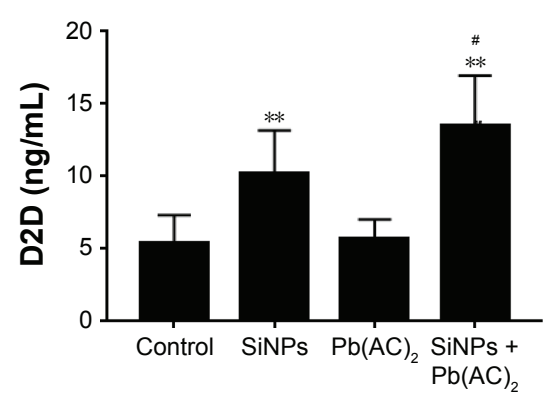

B

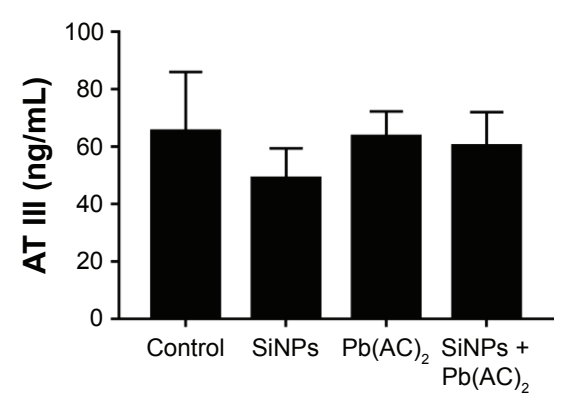

C

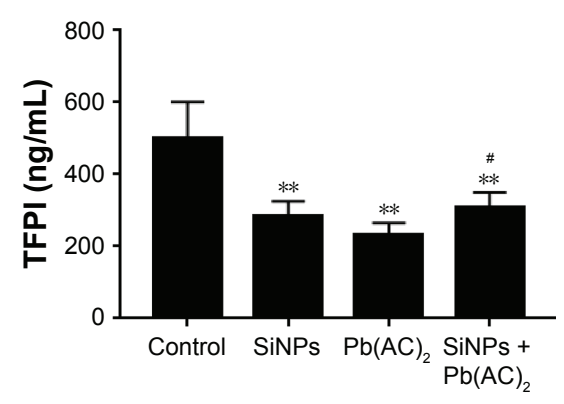

D

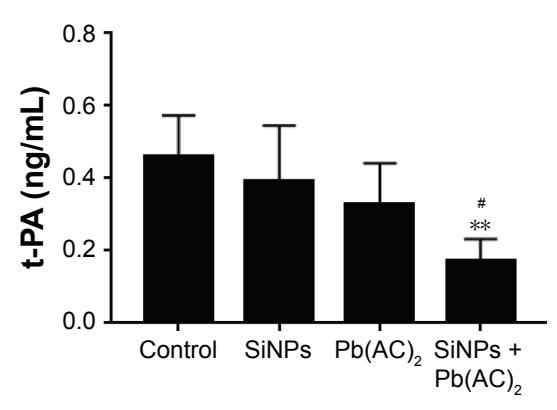

E

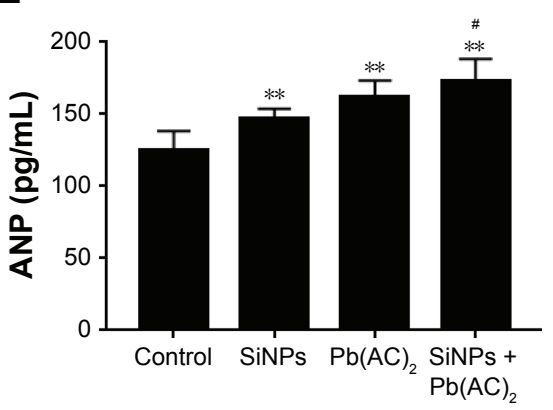

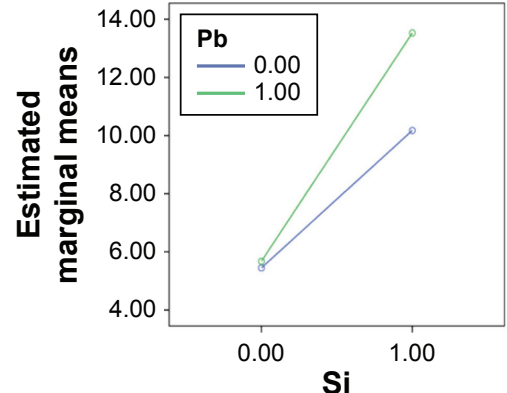
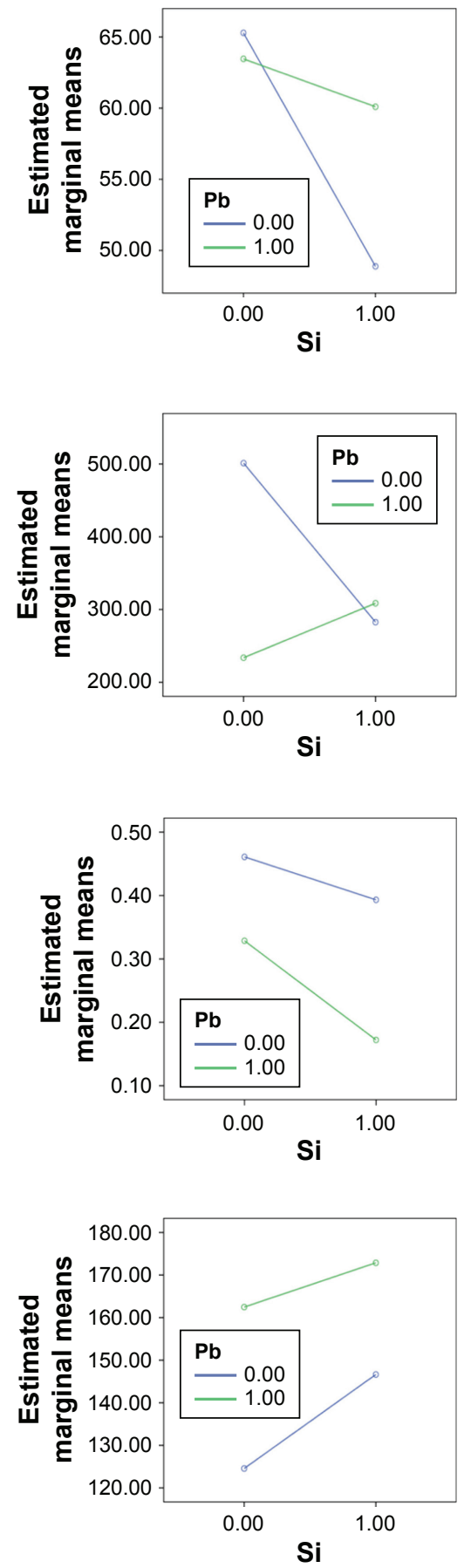

Figure 6 (Continued) 

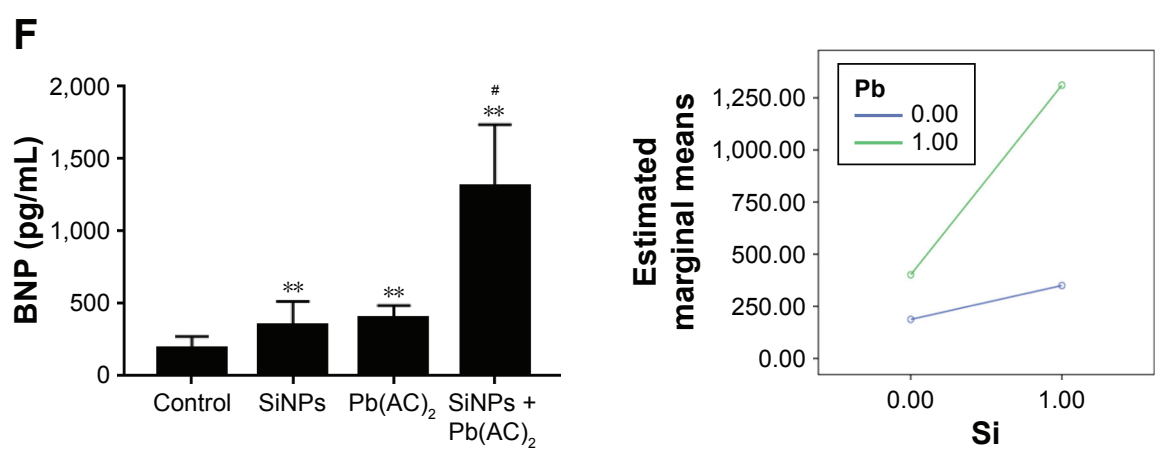

Figure 6 Coagulate- and myocardial-related factors.

Notes: (A) D2D results showed additive interaction between $\mathrm{SiNPs}$ and $\mathrm{Pb}(\mathrm{AC})_{2}(\mathrm{~F}=2.229, \mathrm{P}=0.15 \mathrm{I})$, ${ }^{*} \mathrm{P}<0.05$ when comparing co-exposure group with control and $\mathrm{SiNPs}$ groups in D2D. (B) AT III results showed additive interaction between SiNPs and $\mathrm{Pb}(\mathrm{AC})_{2}(F=1.337, P=0.26 \mathrm{I})$. (C) TFPI results showed synergistic interaction between $\mathrm{SiNPs}$ and $\mathrm{Pb}(\mathrm{AC})_{2}(\mathrm{~F}=35.640, \mathrm{P}=0.000),{ }^{*} \mathrm{P}<0.05$ when comparing co-exposure group with control and $\mathrm{Pb}(\mathrm{AC})_{2}$ groups in TFPI. (D) t-PA results showed additive interaction between SiNPs and $\mathrm{Pb}(\mathrm{AC})_{2}$ ( $\left.F=0.91 \mathrm{I}, \mathrm{P}=0.35 \mathrm{I}\right),{ }^{\#} \mathrm{P}<0.05$ when comparing co-exposure group with control, $\mathrm{SiNPs}$, and $\mathrm{Pb}(\mathrm{AC})_{2}$ groups in t-PA. (E) ANP results showed additive interaction between $\mathrm{SiNPs}$ and $\mathrm{Pb}(\mathrm{AC})_{2}(F=1.358, P=0.258)$, ${ }^{\# P}<0.05$ when comparing co-exposure group with control and SiNPs groups in ANP. (F) BNP results showed synergistic interaction between $\mathrm{SiNPs}$ and $\mathrm{Pb}(\mathrm{AC})_{2}(\mathrm{~F}=\mid \mathrm{4} .836, \mathrm{P}=0.00 \mathrm{I}),{ }^{\# P}<0.05$ when comparing co-exposure group with control, $\mathrm{SiNPs}$, and $\mathrm{Pb}$ groups in $\mathrm{BNP}$. Data are expressed as mean \pm SD from six independent experiments. ${ }^{*} * P<0.01$ compared with untreated control, ${ }^{*} P<0.05$ compared with individual toxicant alone treated group. Abbreviations: ANP, atrial natriuretic peptide; BNP, type B natriuretic peptide; D2D, D-dimer; AT III, antithrombin III; Pb(AC), lead acetate; SiNPs, silica nanoparticles; TFPI, tissue factor pathway inhibitor; t-PA, tissue-type plasminogen activator.

The number of positive ions (like $\mathrm{Na}^{+}, \mathrm{H}^{+}$) and negative ions (like $\mathrm{Cl}^{-}, \mathrm{OH}^{-}$) will be more in normal saline than in distilled water which has only $\mathrm{H}^{+}$and $\mathrm{OH}^{-}$ions. The interaction between the ions alters the surface charge of the nanomaterials. According to our histopathological manifestation, presented in Figure 2, SiNPs or $\mathrm{Pb}$ damaged the structure of myocardial and endothelial cells mildly, while the low-dose co-exposure group showed more inflammation and injury to heart and blood vessels than that shown by single-exposure group. The intima of endothelium is the most sensitive part

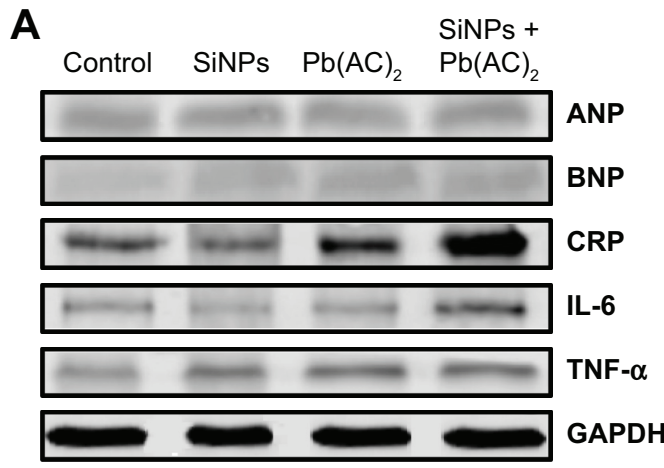

B

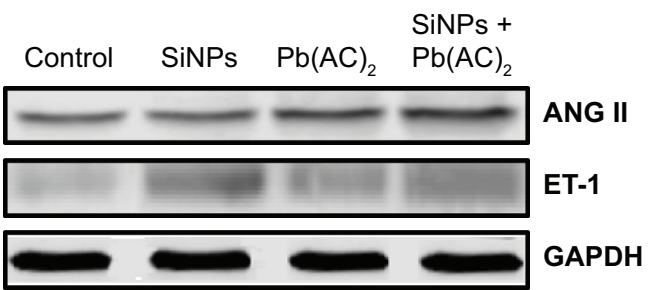

a

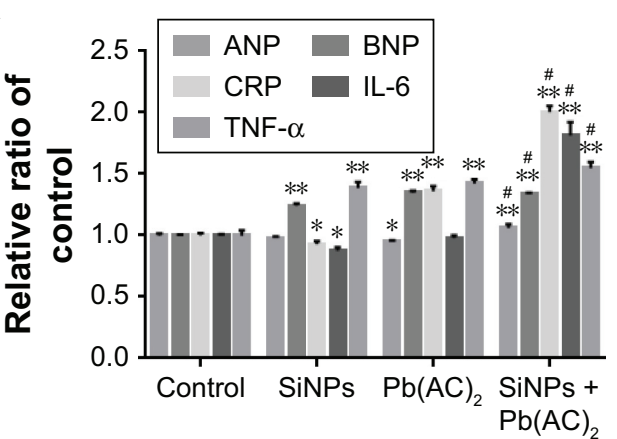

b

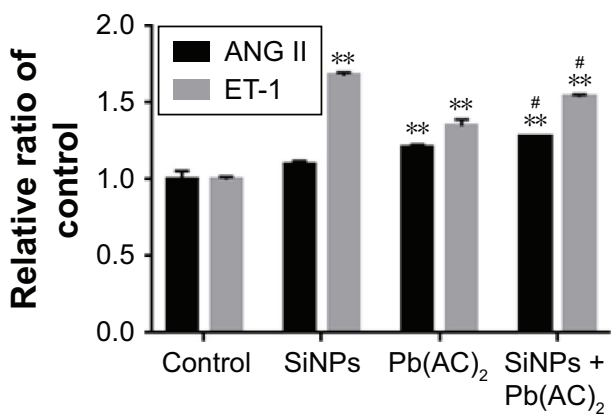

Figure 7 Western-blot assay showing the relative expression of cardiovascular-related proteins.

Notes: (A) Effect on the expression of proteins ANP, BNP, CRP, IL-6, and TNF- $\alpha$ in heart. (a) The gray intensity of related protiens were analyzed by Image J. ${ }^{P}<0.05$ when comparing co-exposure group with control, SiNPs, and Pb groups in ANP, BNP, CRP, IL-6, and TNF- $\alpha$ with the tissue of heart. (B) Effect on the expression of proteins ANG II and ET-I in vessel. (b) The gray intensity of related protiens were analyzed by Image J. ${ }^{P}<0.05$ when comparing co-exposure group with control, SiNPs, and Pb groups in ANG-II and ET-I with the tissue of vessel. GAPDH was used as a control to monitor for equal loading. Data are expressed as mean \pm SD from six independent experiments. ${ }^{*} P<0.05,{ }^{*} * P<0.01$ compared with untreated control, ${ }^{*} P<0.05$ compared with individual toxicant alone treated group.

Abbreviations: ANG II, angiotensin II; ANP, atrial natriuretic peptide; BNP, type B natriuretic peptide; CRP, C-reactive protein; IL-6, interleukin-6; ET-I, endothelin; $\mathrm{GAPDH}$, glyceraldehyde 3-phosphate dehydrogenase; $\mathrm{Pb}(\mathrm{AC})_{2}$, lead acetate; SiNPs, silica nanoparticles. 
to be damaged during the co-exposure. SiNPs have emerged as a promising therapy vector for the heart diseases. SiNPs could also cause typical malformations including pericardial edema and yolk sac edema tail in embryos, resulting in alterations to the normal morphology. ${ }^{26}$ Also, SiNPs affect vascular dysfunction, and the nanotoxicity effect depends on particle size, dose, and cell type. ${ }^{27}$ It is well-known that the endothelium is surrounded by pericytes that communicate with each other. The interactions between endothelial cells directly modulate microvascular development, stabilization, maturation, and remodeling. ${ }^{28}$ SiNPs could be uptaken by the cells which in turn causes inflammation due to the production of inflammatory factors; this further results in the injury to vascular system physically and chemically. ${ }^{29}$ Blood toxicity in SiNPs-induced mice results in oxidative stress damage which was shown to be the reason for their noxious effect. ${ }^{30}$ In rat cardiomyocyte (H9c2) cell line, SiNPs also reduced gap junction intercellular communication, which is one of the targets for biological effects through upregulation of $\mathrm{p}-\mathrm{Cx} 43 .{ }^{31}$ Thus, cardiovascular toxicity of nanoparticles is mainly manifested as inflammation and oxidative stress.

In addition, several studies had verified the cardiovascular toxicity induced by $\mathrm{Pb}$. According to our blood analysis results presented in Figures $3-5, \mathrm{~Pb}$ has shown obvious blood toxicity. Exposure to $\mathrm{Pb}$ accelerated the blood coagulation process and resulted in the hypercoagulation activity. Findings suggested that children intaking higher $\mathrm{Pb}$ concentrations were prone to abnormal measure of cardiovascular physiology, namely abnormal blood pressure, signifying impaired function in cardiovascular system. ${ }^{32} \mathrm{~Pb}$ could increase the expression level of histone acetylation and induce apoptosis in vascular and myocardial tissues. In the early phase of cell cycle, $\mathrm{Pb}$ had been shown to induce growth arrest in cultured rat aorta smooth muscle cells at a high concentration $(500 \mu \mathrm{M})$. $\mathrm{Pb}$ could promote cardiac cell apoptosis and proliferation of the cardiac tissue and cause cardiac cell hypertrophy. ${ }^{33}$ In the event of endothelial layer disruption, $\mathrm{Pb}$ can reach and accumulate in smooth muscle cells, and aggravate inflammation and lipid peroxidation, eventually triggering atherosclerosis. ${ }^{34}$ Lead treatment promoted the activation of NKA and $\mathrm{K}^{+}$channels, and these effects might contribute to the preservation of aortic endothelial function against oxidative stress. ${ }^{35}$ Therefore, exposure to $\mathrm{Pb}$ mainly manifested in the myocardial muscle dysfunction and vasofunctional disturbances.

Furthermore, our co-exposure model results indicated both additive and synergistic effects on blood toxicity. AST is often used as the marker to evaluate the myocardial enzyme function. The CHO, TG, HDL-C and LDL-C levels are used as the markers to evaluate blood lipid levels. Plasma HDL-C levels are inversely related to cardiovascular disease risk. ${ }^{36}$ But the results showed no significant transformation (Figure 4). The possible reason might be that the low-dose exposure to single pollutant might stimulate the mechanism of body protection which could resist the hazard of toxicants. Current studies show that the lipid level could increase after SiNPs exposure; however, we did not find any change in the lipid level in our research. The possible reason may be related to the low concentration of exposure or might activate some pathway of glycolipid metabolism. The underlying mechanism needs to be explored deeply in further studies. Erythrocyte hemolysis and platelet aggregation were induced by the administration of SiNPs and $\mathrm{Pb}$. Besides, many epidemiology studies and animal tests indicated the effects induced by SiNPs or Pb on blood system. Nanoparticles could disturb the stability of the coagulation system, change the hemorheology, and promote the hypercoagulability before thermogenesis. SiNPs penetrated the platelet plasma membrane and stimulated a rapid and prolonged NO release, and finally resulted in the platelet aggregation. ${ }^{37}$

Amorphous SiNPs are able to increase the activation of coagulation cascade by adsorbing and stimulating the intrinsic pathway coagulation factors which in turn result in shortening of coagulation time in APTT and PT tests, as well as enhancing the factor $\mathrm{X}$ activation by russell's viper venom (RVV) in blood plasma but not in the sample with removed factors XI and XII. ${ }^{38}$ SiNPs could elevate the level of FIB, increase the blood viscosity, shorten the time of PT test, and thus increase the risk of thermogenesis, the findings which are consistent with our study results (Figure 5). ${ }^{39}$ TFPI is an inhibitor of extrinsic pathway. TFPI were decreased which showed the activation of extrinsic pathway of blood coagulation. D-dimer is a marker of fibrinolysis system. Increased D-dimer levels activated the promoter of anticoagulative system, as shown in Figure 6. In addition, $\mathrm{Pb}$ could also cause changes in the blood system. Both acute and chronic $\mathrm{Pb}$ exposure has potential to cause many deleterious systematic effects, including hypertension, frank anemia, cognitive deficits, infertility, immune imbalances, delayed skeletal and deciduous dental development, vitamin D deficiency, and gastrointestinal effects. ${ }^{40}$ Acute exposure to low $\mathrm{Pb}$ concentrations produced direct positive inotropic and lusitropic effects on myocardial contractility and increased the right and left ventricular systolic pressure, potentially contributing to the early development of hypertension. ${ }^{41}$ Hypercoagulable state was liable to induce hypertensive disorder and disseminate intravascular coagulation. $\mathrm{Pb}$ could 
increase the levels of alkline phosphatase (AKP), acyl carrier protein (ACP), albumin (ALB), and total protein (TP) significantly and decrease the level of hemoglobin (HGB). ${ }^{42}$ Lead-induced oxidative stress as an underlying mechanism for a plastic anemia warrants further research. ${ }^{43}$

The Western blot results (Figure 7) indicated the increased expressions of ANP, BNP, CRP, IL-6, and TNF- $\alpha$ in coexposure group. SiNPs could stimulate the co-culture of human umbilical vein endothelial cells and monocytes, and increase the expression of TNF- $\alpha$, IL- $1 \beta$, and inflammatory cytokine IL-6 significantly in our previous study. ${ }^{44}$ SiNPs could increase oxidative stress and lead to mitochondrial dysfunction and low energy status. $\mathrm{Pb}$ exposure could reduce the level of nitric oxide, increase the level of ET-1, and inhibit the activity of $\mathrm{Na}^{+} / \mathrm{K}^{+}$-ATPase, consistent with the results of our study. SiNPs could increase the intracellular level of mitochondrial ROS, eventually resulting in the collapse of mitochondrial membrane potential and impairment in ATP synthesis, cellular respiration, and the activities of three ATPdependent enzymes (including $\mathrm{Na}^{+} / \mathrm{K}^{+}$-ATPase, $\mathrm{Ca}^{2+}$-ATPase, and $\mathrm{Ca}^{2+} / \mathrm{Mg}^{2+}$-ATPase), as well as an elevated intracellular calcium level. ${ }^{45}$ In this study, the expression of ANG II and ET-1 was increased. ANG II and ET-1 are strong vasoconstrictors that may raise blood pressure and result in possible injury to blood vessels. Therefore, the co-exposure toxicity of SiNPs and $\mathrm{Pb}$ at low doses mainly involved additive and synergistic interactions in the cardiovascular system. However, more additional evidence about the blood coagulation function of nanoparticles and heavy metals is still required.

According to the previous research, studies on toxicity due to co-exposure to SiNPs and $\mathrm{Pb}$ in humans are limited. But we could easily find that the individual toxicity of SiNPs or $\mathrm{Pb}$ in human had ordered more cardiovascular toxicity. Silica exposure during cigarette smoking elevated the proportion of mortality and accounted for $35.7 \%$ of cardiovascular disease. ${ }^{46}$ Swedish foundry workers exposed to respirable silica exhibited elevated morbidity and mortality from stroke, but not from myocardial infarction, and an obvious relationship between silica exposure and morbidity from stroke was found at a younger age than among the general population. ${ }^{47}$ In addition, increased production of inflammatory cells and mediators, including cytokines and chemokines, due to lead exposure was suggested to be the main cause for various organ disorders..$^{48}$ Cardiovascular toxicity of lead manifested primarily as an effect on blood pressure and eventually increased the risk of atherosclerosis and cardiovascular events. Serum biomarkers including higher lipid profiles, inflammatory cytokines, plasma lipoprotein-associated phospholipase A2, and lower HDL levels were observed with lead. Concomitant abnormal measures of cardiovascular physiology result in an augmented prevalence of vascular inflammation, as well as lipid disorders. ${ }^{49}$ Previous epidemiological and laboratory studies on humans had indicated the cardiovascular toxicity of silica or lead, which is consistent with our study. It is a wellknown fact that there are many kinds of organic and inorganic components in the air. As constituents of air, nanoparticles and numerous metals undergo complex chemical reactions and can cause multisystem diseases. Since the studies on the effects of co-exposure of nanoparticles and heavy metals were limited, effect of co-exposure toxicity on cardiovascular system needed more wide attention, which was the essence of this study. These findings will provide persuasive evidence not only for the safety evaluation of nanoparticles but also for the low-dose combined toxicity on the cardiovascular system.

\section{Conclusion}

In summary, the co-exposure of SiNPs and $\mathrm{Pb}$ at low doses in vivo demonstrated that the additive and synergistic effects are the major interactions that cause inflammation response, hypercoagulation, and cardiac injury, which were liable to trigger the cardiovascular disease. This work has successfully established the interactive effects resulting from co-exposure to nanoparticles and pollutants and offered the biological evidences for their toxicity on cardiovascular system in vivo.

\section{Acknowledgment}

This work was supported by National Natural Science Foundation of China (No. 81230065, 81571130090) and Young Talent Supporting Engineering of China Association for Science and Technology (2017QNRC001).

\section{Disclosure}

The authors report no conflicts of interest in this work.

\section{References}

1. Halas NJ, Naomi JH. Nanoscience under glass: the versatile chemistry of silica nanostructures. ACS Nano. 2008;2(2):179-183.

2. Lu X, Tian Y, Zhao Q, Jin T, Xiao S, Fan X. Integrated metabonomics analysis of the size-response relationship of silica nanoparticles-induced toxicity in mice. Nanotechnology. 2011;22(5):055101.

3. Yu Y, Duan J, Li Y, et al. Combined toxicity of amorphous silica nanoparticles and methylmercury to human lung epithelial cells. Ecotoxicol Environ Saf. 2015;112:144-152.

4. Lu CF, Yuan XY, Li LZ, et al. Combined exposure to nano-silica and lead induced potentiation of oxidative stress and DNA damage in human lung epithelial cells. Ecotoxicol Environ Saf. 2015;122:537-544.

5. Lu CF, Li LZ, Zhou W, Zhao J, Wang YM, Peng SQ. Silica nanoparticles and lead acetate co-exposure triggered synergistic cytotoxicity in A549 cells through potentiation of mitochondria-dependent apoptosis induction. Environ Toxicol Pharmacol. 2017;52:114-120.

6. Du Z, Zhao D, Jing L, et al. Cardiovascular toxicity of different sizes amorphous silica nanoparticles in rats after intratracheal instillation. Cardiovasc Toxicol. 2013;13(3):194-207. 
7. Lee J, Freeman JL. Zebrafish as a model for investigating developmental lead $(\mathrm{Pb})$ neurotoxicity as a risk factor in adult neurodegenerative disease: a mini-review. Neurotoxicology. 2014;43:57-64.

8. Bollati V, Baccarelli A. Environmental epigenetics. Heredity. 2010;105(1): 105-112.

9. Silva MA, de Oliveira TF, Almenara CC, et al. Exposure to a Low Lead Concentration Impairs Contractile Machinery in Rat Cardiac Muscle. Biol Trace Elem Res. 2015;167(2):280-287.

10. Abd-Elhakim YM, El Sharkawy NI, El Bohy KM, Gomaa M, Haseeb S, Morphological HS. Morphological, biochemical, and histopathological postmortem ocular indices following subchronic exposure to cadmium and/or lead in a rabbit model. Environ Sci Pollut Res Int. 2018;25(7): 6619-6632.

11. Guo J, Shi T, Cui X, et al. Effects of silica exposure on the cardiac and renal inflammatory and fibrotic response and the antagonistic role of interleukin-1 beta in C57BL/6 mice. Arch Toxicol. 2016;90(2): 247-258.

12. You R, Ho YS, Hung CH, et al. Silica nanoparticles induce neurodegeneration-like changes in behavior, neuropathology, and affect synapse through MAPK activation. Part Fibre Toxicol. 2018;15(1):28.

13. Higashisaka K, Nakashima A, Iwahara Y, et al. Neutrophil Depletion Exacerbates Pregnancy Complications, Including Placental Damage, Induced by Silica Nanoparticles in Mice. Front Immunol. 2018;9:9.

14. Hou L, Zheng Y, Wang Y, et al. Self-Regulated Carboxyphenylboronic Acid-Modified Mesoporous Silica Nanoparticles with "Touch Switch" Releasing Property for Insulin Delivery. ACS Appl Mater Interfaces. 2018;10(26):21927-21938.

15. Sun L, Li Y, Liu X, et al. Cytotoxicity and mitochondrial damage caused by silica nanoparticles. Toxicol In Vitro. 2011;25(8):1619-1629.

16. Ennos R. Statistical and Data Handling Skills in Biology. 2nd ed. London, UK: Pearson Education Limited; 2007:55-66.

17. Yu Y, Duan J, Li Y, et al. Combined toxicity of amorphous silica nanoparticles and methylmercury to human lung epithelial cells. Ecotoxicol Environ Saf. 2015;112:144-152.

18. Lu CF, Yuan XY, Li LZ, et al. Combined exposure to nano-silica and lead induced potentiation of oxidative stress and DNA damage in human lung epithelial cells. Ecotoxicol Environ Saf. 2015;122:537-544.

19. Jiang J, Oberdörster G, Biswas P. Characterization of size, surface charge, and agglomeration state of nanoparticle dispersions for toxicological studies. J Nanopart Res. 2009;11(1):77-89.

20. Guerrero-Beltrán CE, Bernal-Ramírez J, Lozano O, et al. Silica nanoparticles induce cardiotoxicity interfering with energetic status and $\mathrm{Ca}^{2+}$ handling in adult rat cardiomyocytes. Am J Physiol Heart Circ Physiol. 2017;312(4):H645-H661.

21. Xu LH, Mu FF, Zhao JH, et al. Lead Induces Apoptosis and Histone Hyperacetylation in Rat Cardiovascular Tissues. PLoS One. 2015; 10(6):e0129091.

22. Asweto $\mathrm{CO}, \mathrm{Wu}, \mathrm{Hu} \mathrm{H}$, et al. Combined effect of silica nanoparticles and benzo[a]pyrene on cell cycle arrest induction and apoptosis in human umbilical vein endothelial cells. Int J Environ Res Public Health. 2017;14(3):E289.

23. Duan J, Yu Y, Li Y, Wang Y, Sun Z. Inflammatory response and blood hypercoagulable state induced by low level co-exposure with silica nanoparticles and benzo[a]pyrene in zebrafish (Danio rerio) embryos. Chemosphere. 2016;151:152-162.

24. Feng L, Yang X, Asweto CO, et al. Low-dose combined exposure of nanoparticles and heavy metal compared with $\mathrm{PM}_{2.5}$ in human myocardial AC16 cells. Environ Sci Pollut Res Int. 2017;24(36): 27767-27777.

25. Wang W, Ding X, Xu Q, Wang J, Wang L, Lou X. Zeta-potential data reliability of gold nanoparticle biomolecular conjugates and its application in sensitive quantification of surface absorbed protein. Colloids Surf B Biointerfaces. 2016;148:541-548.

26. Duan J, Yu Y, Shi H, et al. Toxic effects of silica nanoparticles on zebrafish embryos and larvae. PLoS One. 2013;8(9):e74606.

27. Blechinger J, Bauer AT, Torrano AA, Gorzelanny C, Bräuchle C, Schneider SW. Uptake kinetics and nanotoxicity of silica nanoparticles are cell type dependent. Small. 2013;9(23):3970-3980.
28. Kotecki M, Zeiger AS, Van Vliet KJ, Herman IM. Calpain- and talindependent control of microvascular pericyte contractility and cellular stiffness. Microvasc Res. 2010;80(3):339-348.

29. Kim JY, Park JH, Kim M, et al. Safety of nonporous silica nanoparticles in human corneal endothelial cells. Sci Rep. 2017;7(1):14566.

30. Shrivastava R, Kushwaha P, Bhutia YC, Flora S. Oxidative stress following exposure to silver and gold nanoparticles in mice. Toxicol Ind Health. 2016;32(8):1391-1404.

31. Du ZJ, Cui GQ, Zhang J, et al. Inhibition of gap junction intercellular communication is involved in silica nanoparticles-induced $\mathrm{H} 9 \mathrm{c} 2$ cardiomyocytes apoptosis via the mitochondrial pathway. Int J Nanomedicine. 2017;12:2179-2188.

32. Lu X, Xu X, Zhang Y, Zhang Y, Wang C, Huo X. Elevated inflammatory Lp-PLA2 and IL-6 link e-waste $\mathrm{Pb}$ toxicity to cardiovascular risk factors in preschool children. Environ Pollut. 2018;234:601-609.

33. Simões MR, Preti SC, Azevedo BF, et al. Low-level Chronic Lead Exposure Impairs Neural Control of Blood Pressure and Heart Rate in Rats. Cardiovasc Toxicol. 2017;17(2):190-199.

34. Di A, Mehta D, Malik AB. ROS-activated calcium signaling mechanisms regulating endothelial barrier function. Cell Calcium. 2016; 60(3):163-171.

35. Fiorim J, Ribeiro RF, Azevedo BF, et al. Activation of $\mathrm{K}+$ channels and $\mathrm{Na}+\mathrm{K}+\mathrm{ATPase}$ prevents aortic endothelial dysfunction in 7-day lead-treated rats. Toxicol Appl Pharmacol. 2012;262(1):22-31.

36. Annema W, Tietge UJ. Role of hepatic lipase and endothelial lipase in high-density lipoprotein-mediated reverse cholesterol transport. Curr Atheroscler Rep. 2011;13(3):257-265.

37. Corbalan JJ, Medina C, Jacoby A, Malinski T, Radomski MW. Amorphous silica nanoparticles aggregate human platelets: potential implications for vascular homeostasis. Int J Nanomedicine. 2012;7:631-639.

38. Gryshchuk V, Galagan N. Silica Nanoparticles Effects on Blood Coagulation Proteins and Platelets. Biochem Res Int. 2016;2016:2959414.

39. Sanfins E, Augustsson C, Dahlbäck B, Linse S, Cedervall T. Sizedependent effects of nanoparticles on enzymes in the blood coagulation cascade. Nano Lett. 2014;14(8):4736-4744.

40. Mitra P, Sharma S, Purohit P, Sharma P. Clinical and molecular aspects of lead toxicity: An update. Crit Rev Clin Lab Sci. 2017;54(7-8):506-528.

41. Fioresi M, Furieri LB, Simões MR, et al. Acute exposure to lead increases myocardial contractility independent of hypertension development. Braz J Med Biol Res. 2013;46(2):178-185.

42. Qian CY, Li L, Gao ZY, Pk L, Jiang RF, Song WM. Pneumotoxicity and Hemato toxicity of Airborne Fine Particles and Lead Compounds on Rats. J Environ Occup Med. 2011;28:20-24.

43. Ahamed M, Akhtar MJ, Verma S, Kumar A, Siddiqui MK. Environmental lead exposure as a risk for childhood aplastic anemia. Biosci Trends. 2011;5(1):38-43.

44. Liu X, Xue Y, Ding T, Sun J. Enhancement of proinflammatory and procoagulant responses to silica particles by monocyte-endothelial cell interactions. Part Fibre Toxicol. 2012;9:36

45. Guo C, Wang J, Jing L, et al. Mitochondrial dysfunction, perturbations of mitochondrial dynamics and biogenesis involved in endothelial injury induced by silica nanoparticles. Environ Pollut. 2018;236:926-936.

46. Lai H, Liu Y, Zhou M, et al. Combined effect of silica dust exposure and cigarette smoking on total and cause-specific mortality in iron miners: a cohort study. Environ Health. 2018;17(1):46.

47. Fan C, Graff P, Vihlborg P, Bryngelsson IL, Andersson L. Silica exposure increases the risk of stroke but not myocardial infarction-A retrospective cohort study. PLoS One. 2018;13(2):e0192840.

48. Boskabady M, Marefati N, Farkhondeh T, Shakeri F, Farshbaf A, Boskabady MH. The effect of environmental lead exposure on human health and the contribution of inflammatory mechanisms, a review. Environ Int. 2018;120:404-420.

49. Lu X, Xu X, Zhang Y, Zhang Y, Wang C, Huo X. Elevated inflammatory Lp-PLA2 and IL-6 link e-waste Pb toxicity to cardiovascular risk factors in preschool children. Environ Pollut. 2018;234:601-609. 


\section{Supplementary materials}

Table SI Factorial design experiments were applied based on the description

\begin{tabular}{l|l|l}
\hline $\mathbf{P b}$ & \multicolumn{2}{|l}{ SiNPs } \\
\cline { 2 - 3 } & - & + \\
\hline- & 0 & $2 \mathrm{mg} / \mathrm{kg} \cdot \mathrm{kw}$ \\
+ & $0.25 \mathrm{mg} / \mathrm{kg} \cdot \mathrm{kw}$ & $0.25 \mathrm{mg} / \mathrm{kg} \cdot \mathrm{kw}+2 \mathrm{mg} / \mathrm{kg} \cdot \mathrm{kw}$ \\
\hline
\end{tabular}

Abbreviations: Pb, lead acetate; SiNPs, silica nanoparticles.

Table S2 Hydrodynamic size and zeta potential of SiNPs and Pb mixture in distilled water and normal saline as dispersion medium at different time points

\begin{tabular}{l|l|l|l|l}
\hline \multirow{2}{*}{$\begin{array}{l}\text { Time } \\
\text { (hours) }\end{array}$} & \multicolumn{2}{|l|}{ Size (nm) } & Zeta (mV) & Normal saline \\
\cline { 2 - 5 } & Distilled water & Normal saline & Distilled water & $-33.17 \pm 2.01$ \\
\hline 0 & $121.07 \pm 0.91$ & $115.80 \pm 0.70$ & $-29.83 \pm 1.33$ & $-27.97 \pm 1.31$ \\
24 & $117.50 \pm 1.06$ & $115.53 \pm 3.60$ & $-28.20 \pm 1.15$ & $-30.97 \pm 0.15$ \\
48 & $114.60 \pm 1.06$ & $117.90 \pm 0.60$ & $-24.07 \pm 1.19$ & \\
\hline
\end{tabular}

Abbreviations: Pb, lead acetate; SiNPs, silica nanoparticles. 


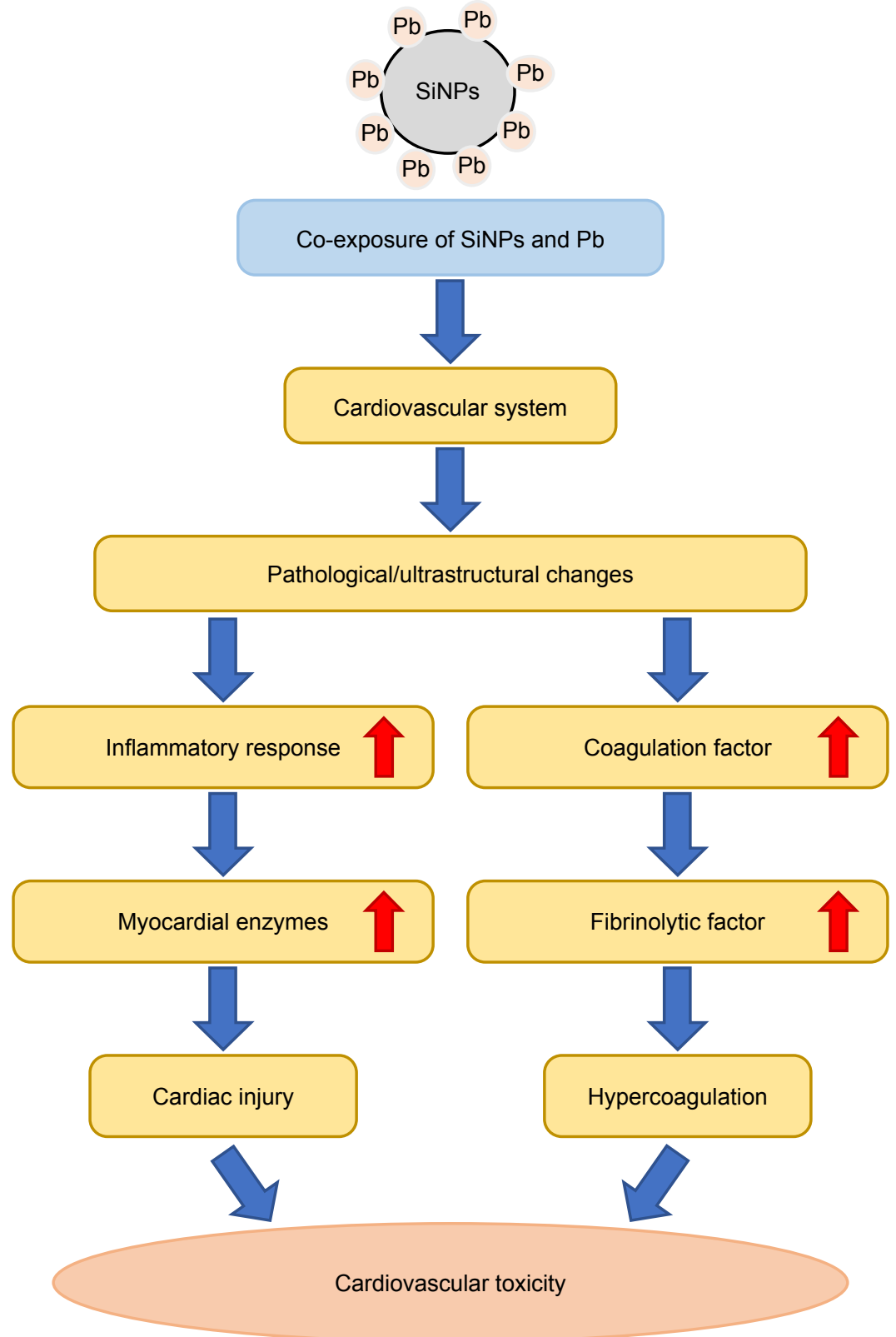

Figure SI The schematic representation of cardiac and blood toxicity induced by co-exposure to SiNPs and Pb.

Abbreviations: $\mathrm{Pb}$, lead; SiNPs, silica nanoparticles.

International Journal of Nanomedicine

Dovepress

\section{Publish your work in this journal}

The International Journal of Nanomedicine is an international, peerreviewed journal focusing on the application of nanotechnology in diagnostics, therapeutics, and drug delivery systems throughout the biomedical field. This journal is indexed on PubMed Central, MedLine, CAS, SciSearch ${ }^{\circledR}$, Current Contents $® /$ Clinical Medicine,

Journal Citation Reports/Science Edition, EMBase, Scopus and the Elsevier Bibliographic databases. The manuscript management system is completely online and includes a very quick and fair peer-review system, which is all easy to use. Visit http://www.dovepress.com/ testimonials.php to read real quotes from published authors.

Submit your manuscript here: http://www.dovepress.com/international-journal-of-nanomedicine-journal 